\title{
Jacobi decomposition of weighted Triebel-Lizorkin and Besov spaces
}

\author{
by
George Kyriazis (Nicosia), Pencho Petrushev (Columbia, SC) and YUAN XU (Eugene, OR)

\begin{abstract}
The Littlewood-Paley theory is extended to weighted spaces of distributions on $[-1,1]$ with Jacobi weights $w(t)=(1-t)^{\alpha}(1+t)^{\beta}$. Almost exponentially localized polynomial elements (needlets) $\left\{\varphi_{\xi}\right\},\left\{\psi_{\xi}\right\}$ are constructed and, in complete analogy with the classical case on $\mathbb{R}^{n}$, it is shown that weighted Triebel-Lizorkin and Besov spaces can be characterized by the size of the needlet coefficients $\left\{\left\langle f, \varphi_{\xi}\right\rangle\right\}$ in respective sequence spaces.
\end{abstract}

1. Introduction. The $\varphi$-transform of Frazier and Jawerth $[6,7,8]$ is a powerful tool for decomposition of spaces of functions or distributions on $\mathbb{R}^{n}$. Our goal in this paper is to develop similar tools for decomposition of weighted spaces of distributions on $[-1,1]$ with Jacobi weights

$$
w(x):=w_{\alpha, \beta}(x):=(1-x)^{\alpha}(1+x)^{\beta}, \quad \alpha, \beta>-1 / 2 .
$$

We will build upon the elements constructed in [13] and termed needlets. The targeted spaces are weighted Triebel-Lizorkin and Besov spaces on $[-1,1]$.

The main vehicle in constructing our building blocks will be the classical Jacobi polynomials $\left\{P_{n}^{(\alpha, \beta)}\right\}_{n=0}^{\infty}$, which form an orthogonal basis for $L^{2}(w):=L^{2}([-1,1], w)$ and are normalized by $P_{n}^{(\alpha, \beta)}(1)=\left(\begin{array}{c}n+\alpha \\ n\end{array}\right)[18]$. In particular,

$$
\int_{-1}^{1} P_{n}^{(\alpha, \beta)}(x) P_{m}^{(\alpha, \beta)}(x) w(x) d x=\delta_{n, m} h_{n}^{(\alpha, \beta)},
$$

where $h_{n}^{(\alpha, \beta)} \sim n^{-1}$ with constants of equivalence depending only on $\alpha$ and $\beta$. Then the normalized Jacobi polynomials $\mathrm{P}_{n}(x)=\mathrm{P}_{n}^{(\alpha, \beta)}(x)$, defined by

$$
\mathrm{P}_{n}(x):=\left(h_{n}^{(\alpha, \beta)}\right)^{-1 / 2} P_{n}^{(\alpha, \beta)}(x), \quad n=0,1, \ldots,
$$

2000 Mathematics Subject Classification: 42A38, 42B08, 42B15.

Key words and phrases: localized polynomial kernels, Jacobi weight, Triebel-Lizorkin spaces, Besov spaces, frames.

The second author has been supported by NSF Grant DMS-0709046 and the third author by NSF Grant DMS-0604056. 
form an orthonormal basis for $L^{2}(w)$, where the inner product is defined by

$$
\langle f, g\rangle:=\int_{-1}^{1} f(x) \overline{g(x)} w(x) d x .
$$

Consequently, for every $f \in L^{2}(w)$,

$$
f=\sum_{n=0}^{\infty} a_{n}(f) \mathrm{P}_{n} \quad \text { with } \quad a_{n}(f):=\left\langle f, \mathrm{P}_{n}\right\rangle .
$$

Then the kernel of the $n$th partial sum operator is

$$
K_{n}(x, y):=\sum_{\nu=0}^{n} \mathrm{P}_{\nu}(x) \mathrm{P}_{\nu}(y) .
$$

Our construction of needlets relies on the fundamental fact [13] that if the coefficients on the right in (1.6) are "smoothed out" by sampling a compactly supported $C^{\infty}$ cut-off function, then the resulting kernel has nearly exponential localization around the main diagonal $y=x$ in $[-1,1]^{2}$. To be more specific, let

$$
L_{n}(x, y):=\sum_{j=0}^{\infty} \widehat{a}\left(\frac{j}{n}\right) \mathrm{P}_{j}(x) \mathrm{P}_{j}(y)
$$

with $\widehat{a}$ admissible in the sense of the following definition:

Definition 1.1. A function $\widehat{a} \in C^{\infty}[0, \infty)$ is said to be admissible of type

(a) if supp $\widehat{a} \subset[0,2]$ and $\widehat{a}(t)=1$ on $[0,1]$, and of type

(b) if supp $\widehat{a} \subset[1 / 2,2]$.

As a companion to the weight $w(x)$ we introduce the quantity

$$
\mathcal{W}(n ; x)=\mathcal{W}_{\alpha, \beta}(n ; x):=\left(1-x+n^{-2}\right)^{\alpha+1 / 2}\left(1+x+n^{-2}\right)^{\beta+1 / 2} .
$$

We will also need the distance on $[-1,1]$ defined by

$$
d(x, y):=|\arccos x-\arccos y| \text {. }
$$

Now one of the main results from [13] can be stated as follows: Let $\widehat{a}$ be admissible. Then for any $\sigma>0$ there is a constant $c_{\sigma}>0$ depending only on $\sigma, \alpha, \beta$, and $\widehat{a}$ such that

$$
\left|L_{n}(x, y)\right| \leq c_{\sigma} \frac{n}{\sqrt{\mathcal{W}(n ; x)} \sqrt{\mathcal{W}(n ; y)}(1+n d(x, y))^{\sigma}}, \quad x, y \in[-1,1] .
$$

The kernels $L_{n}(x, y)$ are the main ingredient in constructing needlet systems here. Our construction utilizes a semidiscrete Calderón type decomposition combined with discretization using the Gaussian quadrature formula (see §3). Earlier in [11] a similar scheme has been used for the construction of frames on the sphere. 
Denoting by $\left\{\varphi_{\xi}\right\}_{\xi \in \mathcal{X}}$ and $\left\{\psi_{\xi}\right\}_{\xi \in \mathcal{X}}$ the constructed analysis and synthesis needlet systems, indexed by a multilevel set $\mathcal{X}=\bigcup_{j=0}^{\infty} \mathcal{X}_{j}$, we show that every distribution $f$ on $[-1,1]\left(f \in \mathcal{D}^{\prime}\right)$ has the representation

$$
f=\sum_{\xi \in \mathcal{X}}\left\langle f, \varphi_{\xi}\right\rangle \psi_{\xi} .
$$

In this article we use the needlets to characterize two scales of weighted Triebel-Lizorkin (F-spaces) and Besov spaces (B-spaces) on $[-1,1]$ defined via Jacobi expansions. The idea of using orthogonal or spectral decompositions for introducing Triebel-Lizorkin and Besov spaces is natural and well known (see $[15,19])$. To be more precise, let

$$
\Phi_{0}(x, y):=\mathrm{P}_{0}(x) \mathrm{P}_{0}(y), \quad \Phi_{j}(x, y):=\sum_{\nu=0}^{\infty} \widehat{a}\left(\frac{\nu}{2^{j-1}}\right) \mathrm{P}_{\nu}(x) \mathrm{P}_{\nu}(y), \quad j \geq 1,
$$

where $\widehat{a}$ is admissible of type (b) (see Definition 1.1) and $\widehat{a}>0$ on $[3 / 5,5 / 3]$.

The first scale of F-spaces $F_{p}^{s q}$ with $s \in \mathbb{R}, 0<p<\infty, 0<q \leq \infty$, is defined $(\S 4)$ as the space of all distributions $f$ on $[-1,1]$ such that

$$
\|f\|_{F_{p}^{s q}}:=\left\|\left(\sum_{j=0}^{\infty}\left(2^{s j}\left|\Phi_{j} * f(\cdot)\right|\right)^{q}\right)^{1 / q}\right\|_{L^{p}(w)}<\infty .
$$

We define a second scale of $F$-spaces $\mathcal{F}_{p}^{s q}(\S 5)$ as the space of all $f \in \mathcal{D}^{\prime}$ such that

$$
\|f\|_{\mathcal{F}_{p}^{s q}}:=\left\|\left(\sum_{j=0}^{\infty}\left[2^{s j} \mathcal{W}\left(2^{j} ; \cdot\right)^{-s}\left|\Phi_{j} * f(\cdot)\right|\right]^{q}\right)^{1 / q}\right\|_{L^{p}(w)}<\infty .
$$

(For the definition of $\Phi_{j} * f$, see (2.32).) The corresponding scales of weighted Besov spaces $B_{p}^{s q}$ (see $[16,19]$ ) and $\mathcal{B}_{p}^{s q}$ with $s \in \mathbb{R}, 0<p, q \leq \infty$, are defined $(\S 6-7)$ via the (quasi-)norms

$$
\|f\|_{B_{p}^{s q}}:=\left(\sum_{j=0}^{\infty}\left(2^{s j}\left\|\Phi_{j} * f\right\|_{L^{p}(w)}\right)^{q}\right)^{1 / q}
$$

and

$$
\|f\|_{\mathcal{B}_{p}^{s q}}:=\left(\sum_{j=0}^{\infty}\left[2^{s j}\left\|\mathcal{W}\left(2^{j} ; \cdot\right)^{-s} \Phi_{j} * f(\cdot)\right\|_{L^{p}(w)}\right]^{q}\right)^{1 / q} .
$$

To some extent the second scales of F- and B-spaces are more natural than the first since they embed correctly with respect to the smoothness parameter $s$ (see $\S 5, \S 7$ for details). Also, the second scale of B-spaces provides the smoothness spaces of nonlinear $n$-term approximation from needlets $(\S 8)$.

One of our main results $(\S 4)$ asserts that for all indices the weighted Triebel-Lizorkin spaces $F_{p}^{s q}$ can be characterized in terms of the size of the 
needlet coefficients, namely,

$$
\|f\|_{F_{p}^{s q}} \sim\left\|\left(\sum_{j=0}^{\infty} 2^{s j q} \sum_{\xi \in \mathcal{X}_{j}}\left|\left\langle f, \varphi_{\xi}\right\rangle \psi_{\xi}(\cdot)\right|^{q}\right)^{1 / q}\right\|_{L^{p}(w)} .
$$

The needlet characterization of the Besov spaces $B_{p}^{s q}(\S 6)$ takes the form

$$
\|f\|_{B_{p}^{s q}} \sim\left(\sum_{j=0}^{\infty} 2^{s j q}\left[\sum_{\xi \in \mathcal{X}_{j}}\left\|\left\langle f, \varphi_{\xi}\right\rangle \psi_{\xi}\right\|_{p}^{p}\right]^{q / p}\right)^{1 / q} .
$$

Characterizations of similar nature are obtained for the second scales of weighted Triebel-Lizorkin and Besov spaces $\mathcal{F}_{p}^{s q}$ and $\mathcal{B}_{p}^{s q}$ (see $\S 5, \S 7$ ). Using $L^{p}(w)$ multipliers we show that the space $F_{p}^{02}=\mathcal{F}_{p}^{02}$ can be identified as $L^{p}(w)$ for $1<p<\infty$.

This is a follow-up paper of [13]; it is closely related to [11] and [9], where needlet decompositions of Triebel-Lizorkin and Besov spaces on the unit sphere and ball are developed.

The rest of the paper is organized as follows. In $\S 2$, some auxiliary facts are given, including localized and reproducing polynomial kernels, Gaussian quadrature, the maximal inequality, and basics of distributions on $[-1,1]$. In $\S 3$, we construct the needlets and show some of their properties. The first and second scales of weighted Triebel-Lizorkin spaces are defined and characterized via neadlets in $\S 4$ and $\S 5$, respectively, while the first and second scales of Besov spaces are defined and characterized via needlets in $\S 6$ and $\S 7$. In $\S 8$, Besov spaces are applied to weighted nonlinear approximation from needlets; a Jackson theorem is proved. Section 9 is an appendix, where the proofs of some statements are given.

Throughout the paper we use the following notation:

$$
\|f\|_{p}:=\left(\int_{-1}^{1}|f(x)|^{p} w(x) d x\right)^{1 / p}, \quad 0<p<\infty, \quad\|f\|_{\infty}:=\sup _{x \in[-1,1]}|f(x)| .
$$

For a measurable set $E \subset[-1,1]$, we set $\mu(E):=\int_{E} w(y) d y$; $\mathbb{1}_{E}$ is the characteristic function of $E$ and $\widetilde{\mathbb{1}}_{E}:=|\mu(E)|^{-1 / 2} \mathbb{1}_{E}$ is the $L^{2}(w)$ normalized characteristic function of $E$. Also, $\Pi_{n}$ denotes the set of all univariate algebraic polynomials of degree $\leq n$. Positive constants are denoted by $c$, $c_{1}, c_{*}, \ldots$ and they may vary at every occurrence. The notation $A \sim B$ means $c_{1} A \leq B \leq c_{2} A$.

\section{Preliminaries}

2.1. Localized kernels induced by Jacobi polynomials. To a large extent our development in this paper relies on the nearly exponential localization (1.10) of kernels $L_{n}(x, y)$ of the form (1.7) with admissible $\widehat{a}$, established in [13]. To avoid some potential confusion, we note that the 
inner product in [13] is defined by $\langle f, g\rangle:=c_{\alpha, \beta} \int_{-1}^{1} f(x) \overline{g(x)} w(x) d x$ with $c_{\alpha, \beta}^{-1}:=\int_{-1}^{1} w(x) d x$ and as a result $L_{n}(x, y)$ from (1.7) is a constant multiple of $L^{\alpha, \beta}(x, y)$ from [13]. A similar remark applies to the constants $h_{n}^{(\alpha, \beta)}$ from (1.2) and [13].

The proof of estimate (1.10) (see [13]) is based on the almost exponential localization of the univariate polynomial:

$$
L_{n}^{\alpha, \beta}(x):=\sum_{j=0}^{\infty} \widehat{a}\left(\frac{j}{n}\right)\left(h_{j}^{(\alpha, \beta)}\right)^{-1} P_{j}^{(\alpha, \beta)}(1) P_{j}^{(\alpha, \beta)}(x) .
$$

Theorem 2.1 ([1, 13]). Assume that $\alpha \geq \beta>-1 / 2$ and let $\widehat{a}$ be admissible. Then for every $k \geq 1$ there exists a constant $c_{k}>0$ depending only on $k, \alpha, \beta$, and $\widehat{a}$ such that

$$
\left|L_{n}^{\alpha, \beta}(\cos \theta)\right| \leq c_{k} \frac{n^{2 \alpha+2}}{(1+n \theta)^{k+\alpha-\beta}}, \quad 0 \leq \theta \leq \pi .
$$

The dependence of $c_{k}$ on $\widehat{a}$ is of the form $c_{k}=c(\alpha, \beta, k) \max _{1 \leq \nu \leq k}\left\|\widehat{a}^{(\nu)}\right\|_{L^{1}}$.

This estimate was proved in [13] with $\widehat{a}$ admissible of type (b) and in [1] with $\widehat{a}$ admissible of type (a) (for a proof, see also [14]).

In [13, Proposition 1] it is shown that (1.10) yields the following upper bound for the weighted $L^{p}$ integrals of $\left|L_{n}(x, y)\right|$ :

$$
\int_{-1}^{1}\left|L_{n}(x, y)\right|^{p} w(y) d y \leq c\left(\frac{n}{\mathcal{W}(n ; x)}\right)^{p-1}, \quad-1 \leq x \leq 1,0<p<\infty
$$

The next theorem shows that in a sense the kernel $L_{n}(x, y)$ from (1.7) is Lip 1 in $x$ (and $y$ ).

TheOrem 2.2. Let $\alpha, \beta>-1 / 2$. Suppose $\widehat{a}$ is admissible and $\sigma>0$ is an arbitrary constant. If $x, y, z, \xi \in[-1,1], d(x, \xi) \leq c_{*} n^{-1}$ and $d(z, \xi) \leq$ $c_{*} n^{-1}$ with $n \geq 1, c_{*}>0$, then

$$
\left|L_{n}(x, y)-L_{n}(\xi, y)\right| \leq c_{\sigma} \frac{n^{2} d(x, \xi)}{\sqrt{\mathcal{W}(n ; y)} \sqrt{\mathcal{W}(n ; z)}(1+n d(y, z))^{\sigma}},
$$

where $c_{\sigma}>0$ depends only on $\sigma, \alpha, \beta, c_{*}$, and $\widehat{a}$.

The proof of this theorem is given in the appendix.

Lower bound estimates for the integrals of $\left|L_{n}(x, y)\right|$ are nontrivial and will be vital for our further development.

Proposition 2.3. Let $\widehat{a}$ be admissible and $|\widehat{a}(t)| \geq c>0$ for $t \in$ $[3 / 5,5 / 3]$. Then

$$
\int_{-1}^{1}\left|L_{n}(x, y)\right|^{2} w(y) d y \geq \operatorname{cn\mathcal {W}}(n ; x)^{-1}, \quad-1 \leq x \leq 1 .
$$


Proof. By the definition of $L_{n}(x, y)$ in (1.7) and the orthogonality of the Jacobi polynomials, it follows that

$$
\int_{-1}^{1}\left|L_{n}(x, y)\right|^{2} w(y) d y \geq c \sum_{k=[n / 2]}^{2 n}|\widehat{a}(k / n)|^{2}\left[\mathrm{P}_{k}^{(\alpha, \beta)}(x)\right]^{2} .
$$

Since $|\widehat{a}(t)| \geq c>0$ for $t \in[3 / 5,5 / 3]$ and $\mathrm{P}_{k}(x)=\left(h_{k}^{(\alpha, \beta)}\right)^{-1 / 2} P_{k}^{(\alpha, \beta)}(x) \sim$ $k^{1 / 2} P_{k}^{(\alpha, \beta)}(x)$, it suffices to prove that

$$
\sum_{k=[3 n / 5]}^{[5 n / 3]}\left[P_{k}^{(\alpha, \beta)}(x)\right]^{2} \geq c \mathcal{W}(n ; x)^{-1}, \quad c>0,
$$

which is established in the following proposition.

Proposition 2.4. If $\alpha, \beta>-1$ and $\varepsilon>0$, then

$$
\Lambda_{n}(x):=\sum_{k=n}^{n+[\varepsilon n]}\left[P_{k}^{(\alpha, \beta)}(x)\right]^{2} \geq c \mathcal{W}(n ; x)^{-1}, \quad x \in[-1,1], n \geq 1 / \varepsilon,
$$

where $c>0$ depending only on $\alpha, \beta$, and $\varepsilon$.

This proposition is nontrivial and its proof is given in the appendix.

2.2. Reproducing kernels and best polynomial approximation. We let $E_{n}(f)_{p}$ denote the best approximation of $f \in L^{p}(w)$ from $\Pi_{n}$, i.e.

$$
E_{n}(f)_{p}:=\inf _{g \in \Pi_{n}}\|f-g\|_{p} .
$$

To simplify our notation we introduce the following "convolution": For functions $\Phi:[-1,1]^{2} \rightarrow \mathbb{C}$ and $f:[-1,1] \rightarrow \mathbb{C}$, we write

$$
\Phi * f(x):=\int_{-1}^{1} \Phi(x, y) f(y) w(y) d y .
$$

Lemma 2.5. Suppose $\widehat{a}$ is admissible of type (a) and let $L_{n}(x, y)$ be the kernel defined in (1.7).

(i) $L_{n}(x, y)$ is a symmetric reproducing kernel for $\Pi_{n}$, i.e. $L_{n} * g=g$ for $g \in \Pi_{n}$.

(ii) For any $f \in L^{p}(w), 1 \leq p \leq \infty$, we have $L_{n} * f \in \Pi_{2 n}$,

$$
\left\|L_{n} * f\right\|_{p} \leq c\|f\|_{p} \quad \text { and } \quad\left\|f-L_{n} * f\right\|_{p} \leq c E_{n}(f)_{p} .
$$

Proof. Part (i) is immediate since $\widehat{a}(\nu / n)=1$ for $0 \leq \nu \leq n$. The lefthand estimate in (2.9) follows from (2.3) when $p=1$ and $p=\infty$; the general case follows by interpolation. The right-hand estimate in (2.9) follows from the left-hand estimate and (i). 
Lemma 2.5(i) and (2.3) are instrumental in proving Nikolski type inequalities.

Proposition 2.6. For $0<q \leq p \leq \infty$ and $g \in \Pi_{n}$,

$$
\|g\|_{p} \leq c n^{(2+2 \min \{0, \max \{\alpha, \beta\}\})(1 / q-1 / p)}\|g\|_{q},
$$

furthermore, for any $s \in \mathbb{R}$,

$$
\left\|\mathcal{W}(n ; \cdot)^{s} g(\cdot)\right\|_{p} \leq c n^{1 / q-1 / p}\left\|\mathcal{W}(n ; \cdot)^{s+1 / p-1 / q} g(\cdot)\right\|_{q}
$$

The proof of this proposition is given in the appendix.

2.3. Quadrature formula and subdivision of $[-1,1]$. For the construction of our building blocks (needlets) we will utilize an appropriate Gaussian quadrature formula. Let $\xi_{j, \nu}=: \cos \theta_{\nu}, \nu=1,2, \ldots, 2^{j+1}$, be the zeros of the Jacobi polynomials $P_{2^{j+1}}^{(\alpha, \beta)}$ ordered so that

$$
0<\theta_{1}<\cdots<\theta_{2^{j+1}}<\pi .
$$

It is well known that uniformly (see [5] and also (9.9)-(9.10) below)

$$
\theta_{\nu+1}-\theta_{\nu} \sim 2^{-j} \text { and hence } \theta_{\nu} \sim \nu 2^{-j} \text {. }
$$

Define now

$$
\mathcal{X}_{j}:=\left\{\xi_{j, \nu}: \nu=1,2, \ldots, 2^{j+1}\right\}, \quad j \geq 0, \quad X:=\bigcup_{j=0}^{\infty} \mathcal{X}_{j} .
$$

As is well known [18] the zeros of the Jacobi polynomial $P_{2^{j+1}}^{(\alpha, \beta)}$ serve as knots of the Gaussian quadrature

$$
\int_{-1}^{1} f(x) w(x) d x \sim \sum_{\xi \in \mathcal{X}_{j}} c_{\xi} f(\xi)
$$

which is exact for all polynomials of degree at most $2^{j+2}-1$. Furthermore, the coefficients $c_{\xi}$ are positive and have the asymptotics

$$
\mathrm{c}_{\xi} \sim \lambda_{2^{j+1}}(\xi) \sim 2^{-j} w(\xi)\left(1-\xi^{2}\right)^{1 / 2} \sim 2^{-j} \mathcal{W}\left(2^{j} ; \xi\right),
$$

where $\lambda_{2^{j+1}}(t)$ is the Christoffel function and the constants of equivalence depend only on $\alpha, \beta$ (cf. e.g. [12]).

We next introduce the $j$ th level weighted dyadic intervals. Set as above $\xi_{j, \nu}=: \cos \theta_{\nu}$ and define

$$
\begin{aligned}
I_{\xi_{j, \nu}} & :=\left[\left(\xi_{j, \nu+1}+\xi_{j, \nu}\right) / 2,\left(\xi_{j, \nu-1}+\xi_{j, \nu}\right) / 2\right], \quad \nu=2,3, \ldots, 2^{j+1}-1, \\
I_{\xi_{j, 1}} & :=\left[\left(\xi_{j, 2}+\xi_{j, 1}\right) / 2,1\right], \quad I_{\xi_{j, 2} j+1}:=\left[-1,\left(\xi_{j, 2^{j+1}}+\xi_{j, 2^{j+1}-1}\right) / 2\right] .
\end{aligned}
$$

For $\xi \in \mathcal{X}_{j}$ we will briefly write $I_{\xi}:=I_{\xi_{j, \nu}}$ if $\xi=\xi_{j, \nu}$.

It follows by (2.12) that there exist constants $c_{1}, c_{2}>0$ such that

$$
B_{\xi}\left(c_{1} 2^{-j}\right) \subset I_{\xi} \subset B_{\xi}\left(c_{2} 2^{-j}\right),
$$


where $B_{y}(r):=\{x \in[-1,1]: d(x, y) \leq r\}$ with $d(\cdot, \cdot)$ being the distance from (1.9). Also, it is straightforward to show that

$$
\mu\left(I_{\xi}\right):=\int_{I_{\xi}} w(x) d x \sim 2^{-j} \mathcal{W}\left(2^{j} ; \xi\right) \sim \mathrm{c}_{\xi}, \quad \xi \in \mathcal{X}_{j}, j \geq 0 .
$$

It will be useful to note that

$$
\begin{array}{ll}
\mathcal{W}(n ; \cos \theta) \sim\left(\sin \theta+n^{-1}\right)^{2 \alpha+1}, & 0 \leq \theta \leq 2 \pi / 3, \\
\mathcal{W}(n ; \cos \theta) \sim\left(\sin \theta+n^{-1}\right)^{2 \beta+1}, & \pi / 3 \leq \theta \leq \pi .
\end{array}
$$

The following simple inequality will be instrumental in various proofs:

$$
\mathcal{W}(n ; x) \leq c \mathcal{W}(n ; y)(1+n d(x, y))^{2 \max \{\alpha, \beta\}+1}, \quad x, y \in[-1,1], n \geq 1 .
$$

For the proof see the appendix.

2.4. The maximal inequality. For every $0<t<\infty$ and $x \in[-1,1]$, we define

$$
\mathcal{M}_{t} f(x):=\sup _{I \ni x}\left(\frac{1}{\mu(I)} \int_{I}|f(y)|^{t} w(y) d y\right)^{1 / t},
$$

where the sup is over all intervals $I \subset[-1,1]$ containing $x$. It is not hard to see that $\mu$ is a doubling measure on $[-1,1]$ and hence the general theory of maximal inequalities applies. In particular the Fefferman-Stein vectorvalued maximal inequality holds (see [17]): If $0<p<\infty, 0<q \leq \infty$ and $0<t<\min \{p, q\}$ then for any sequence of functions $\left\{f_{\nu}\right\}_{\nu=1}^{\infty}$ on $[-1,1]$,

$$
\left\|\left(\sum_{\nu=1}^{\infty}\left|\mathcal{M}_{t} f_{\nu}(\cdot)\right|^{q}\right)^{1 / q}\right\|_{p} \leq c\left\|\left(\sum_{\nu=1}^{\infty}\left|f_{\nu}(\cdot)\right|^{q}\right)^{1 / q}\right\|_{p} .
$$

We need to estimate $\left(\mathcal{M}_{t} \mathbb{1}_{I_{\xi}}\right)(x)$ for the intervals $I_{\xi}$ from $(2.16)-(2.17)$ and other intervals.

Lemma 2.7. Let $\eta \in[0,1]$ and $0<\varepsilon \leq \pi$. Then for $x \in[-1,1]$,

$$
\left(\mathcal{M}_{t} \mathbb{1}_{B_{\eta}(\varepsilon)}\right)(x) \sim\left(1+\frac{d(\eta, x)}{\varepsilon}\right)^{-1 / t}\left(1+\frac{d(\eta, x)}{\varepsilon+d(\eta, 1)}\right)^{-(2 \alpha+1) / t}
$$

and hence

$$
c^{\prime}\left(1+\frac{d(\eta, x)}{\varepsilon}\right)^{-(2 \alpha+2) / t} \leq\left(\mathcal{M}_{t} \mathbb{1}_{B_{\eta}(\varepsilon)}\right)(x) \leq c\left(1+\frac{d(\eta, x)}{\varepsilon}\right)^{-1 / t} .
$$

Here the constants depend only on $\alpha, \beta$, and $t$.

A similar lemma holds for $\eta \in[-1,0)$. We relegate the proof of this lemma to the appendix. 
2.5. Distributions on $[-1,1]$. Here we give some basic and well known facts about distributions on $[-1,1]$. We will use as test functions the set $\mathcal{D}:=C^{\infty}[-1,1]$ of all infinitely differentiable complex-valued functions on $[-1,1]$, where the topology is induced by the seminorms

$$
|\phi|_{k}:=\left\|\phi^{(k)}(t)\right\|_{\infty}, \quad k=0,1, \ldots
$$

Note that the Jacobi polynomials $\left\{\mathrm{P}_{n}\right\}$ belong to $\mathcal{D}$. More importantly, the space $\mathcal{D}$ of test functions $\phi$ can be completely characterized by the coefficients of their Jacobi expansions: $a_{n}(\phi):=\left\langle\phi, \mathrm{P}_{n}\right\rangle:=\int_{-1}^{1} \phi(x) \mathrm{P}_{n}(x) w(x) d x$. Define

$$
\mathcal{N}_{k}(\phi):=\sup _{n \geq 0}(n+1)^{k}\left|a_{n}(\phi)\right| .
$$

Lemma 2.8. (a) $\phi \in \mathcal{D}$ if and only if $a_{n}(\phi)=\mathcal{O}\left(n^{-k}\right)$ for all $k$.

(b) For every $\phi \in \mathcal{D}$ we have $\phi=\sum_{n=0}^{\infty} a_{n}(\phi) \mathrm{P}_{n}$, where the convergence is in the topology of $\mathcal{D}$.

(c) The topology in $\mathcal{D}$ can be equivalently defined by the norms $\mathcal{N}_{k}(\cdot)$, $k=0,1, \ldots$

Proof. If $\phi \in \mathcal{D}$, then due to the orthogonality of $\mathrm{P}_{n}$ to $\Pi_{n-1}$, we have for $n=1,2, \ldots$,

$$
\left|a_{n}(\phi)\right|=\left|\left\langle\phi, \mathrm{P}_{n}\right\rangle\right|=\left|\left\langle\phi-Q_{n-1}, \mathrm{P}_{n}\right\rangle\right| \leq E_{n-1}(\phi)_{2} \leq c_{k} n^{-k}\left\|\phi^{(k)}\right\|_{\infty},
$$

where $Q_{n-1} \in \Pi_{n-1}$ is the polynomial of best $L^{2}(w)$ approximation to $\phi$. Here we used a simple Jackson estimate for approximation from algebraic polynomials $\left(E_{n}(\phi)_{\infty} \leq c_{k} n^{-k}\left\|\phi^{(k)}\right\|_{\infty}\right)$. Therefore, $a_{n}(\phi)=\mathcal{O}\left(n^{-k}\right)$ and $\mathcal{N}_{k}(\phi) \leq c_{k}\|\phi\|_{k}$ for $k=0,1, \ldots$

On the other hand, by Markov's inequality it follows that

$$
\left\|\mathrm{P}_{n}^{(k)}\right\|_{L^{\infty}[-1,1]} \leq n^{2 k}\left\|\mathrm{P}_{n}\right\|_{L^{\infty}[-1,1]} \leq c n^{2 k} h_{n}^{-1 / 2} P_{n}(1) \leq c n^{2 k+\alpha+1 / 2} .
$$

Hence, if $a_{n}(\phi)=\mathcal{O}\left(n^{-k}\right)$ for all $k$, then $\phi^{(k)}=\sum_{n=0}^{\infty} a_{n}(\phi) \mathrm{P}_{n}^{(k)}$ with the series converging uniformly and

$$
|\phi|_{k} \leq c \sum_{n=0}^{\infty}\left|a_{n}(\phi)\right|(n+1)^{2 k+\alpha+1 / 2} \leq c \mathcal{N}_{2 k+[\alpha+1 / 2]+1}(\phi), \quad k=0,1, \ldots,
$$

which completes the proof of the lemma.

The space $\mathcal{D}^{\prime}:=\mathcal{D}^{\prime}[-1,1]$ of distributions on $[-1,1]$ is defined as the set of all continuous linear functionals on $\mathcal{D}$. The pairing of $f \in \mathcal{D}^{\prime}$ and $\phi \in \mathcal{D}$ will be denoted by $\langle f, \phi\rangle:=f(\bar{\phi})$, which will be shown to be consistent with the inner product $\langle f, g\rangle:=\int_{-1}^{1} f(x) \overline{g(x)} w(x) d x$ in $L^{2}(w)$. We will need the representation of distributions from $\mathcal{D}^{\prime}$ in terms of Jacobi polynomials. 
Lemma 2.9. (a) A linear functional $f$ on $\mathcal{D}$ is a distribution $\left(f \in \mathcal{D}^{\prime}\right)$ if and only if there exists $k \geq 0$ such that

$$
|\langle f, \phi\rangle| \leq c_{k} \mathcal{N}_{k}(\phi) \quad \text { for all } \phi \in \mathcal{D},
$$

For $f \in \mathcal{D}^{\prime}$, denote $a_{n}(f):=\left\langle f, \mathrm{P}_{n}\right\rangle$. Then for some $k \geq 0$,

$$
\left|\left\langle f, \mathrm{P}_{n}\right\rangle\right| \leq c_{k}(n+1)^{k}, \quad n=0,1, \ldots
$$

(b) Every $f \in \mathcal{D}^{\prime}$ has the representation $f=\sum_{n=0}^{\infty} a_{n}(f) \mathrm{P}_{n}$ in distributional sense, i.e.

$$
\langle f, \phi\rangle=\sum_{n=0}^{\infty} a_{n}(f)\left\langle\mathrm{P}_{n}, \phi\right\rangle=\sum_{n=0}^{\infty} a_{n}(f) a_{n}(\bar{\phi}) \quad \text { for all } \phi \in \mathcal{D},
$$

where the series converges absolutely.

Proof. (a) Part (a) follows immediately from the fact that the topology in $\mathcal{D}$ can be defined by the norms $\mathcal{N}_{k}(\cdot)$ defined in (2.28).

(b) Using Lemma 2.8(b) we get, for $\phi \in \mathcal{D}$,

$$
\langle f, \phi\rangle=\lim _{N \rightarrow \infty}\left\langle f, \sum_{n=0}^{N} a_{n}(\phi) \mathrm{P}_{n}\right\rangle=\lim _{N \rightarrow \infty} \sum_{n=0}^{N} a_{n}(\bar{\phi})\left\langle f, \mathrm{P}_{n}\right\rangle=\sum_{n=0}^{\infty} a_{n}(f) a_{n}(\bar{\phi}),
$$

where for the last equality we used (2.30) and the fact that $a_{n}(\bar{\phi})$ are rapidly decaying.

It is convenient to extend the "convolution" from (2.8) to the case of distributions.

Definition 2.10. Assuming that $f \in \mathcal{D}^{\prime}$ and $\Phi:[-1,1]^{2} \rightarrow \mathbb{C}$ is such that $\Phi(x, y)$ belongs to $\mathcal{D}$ as a function of $y(\Phi(x, \cdot) \in \mathcal{D})$, we define $\Phi * f$ by

$$
\Phi * f(x):=\langle f, \overline{\Phi(x, \cdot)}\rangle,
$$

where on the right $f$ acts on $\overline{\Phi(x, y)}$ as a function of $y$.

3. Construction of building blocks (needlets). Following the ideas from [13] we next construct two sequences of companion "analysis" and "synthesis" needlets. Our construction is based on a Calderón type reproducing formula. Let $\widehat{a}, \widehat{b}$ satisfy the conditions

$$
\begin{aligned}
& \widehat{a}, \widehat{b} \in C^{\infty}[0, \infty), \quad \text { supp } \widehat{a}, \widehat{b} \subset[1 / 2,2], \\
& |\widehat{a}(t)|,|\widehat{b}(t)|>c>0 \quad \text { if } t \in[3 / 5,5 / 3], \\
& \widehat{\widehat{a}(t)} \widehat{b}(t)+\overline{\widehat{a}}(2 t) \widehat{b}(2 t)=1 \quad \text { if } t \in[1 / 2,1] .
\end{aligned}
$$

Hence,

$$
\sum_{\nu=0}^{\infty} \overline{\widehat{a}\left(2^{-\nu} t\right)} \widehat{b}\left(2^{-\nu} t\right)=1, \quad t \in[1, \infty)
$$


It is easy to show that if $\widehat{a}$ satisfies (3.1)-(3.2), then there exists $\widehat{b}$ satisfying (3.1)-(3.2) such that (3.3) holds true (see e.g. [7]).

Assuming that $\widehat{a}, \widehat{b}$ satisfy $(3.1)-(3.3)$, we define $\Phi_{0}(x, y)=\Psi_{0}(x, y):=$ $\mathrm{P}_{0}(x) \mathrm{P}_{0}(y)$,

$$
\begin{aligned}
& \Phi_{j}(x, y):=\sum_{\nu=0}^{\infty} \widehat{a}\left(\frac{\nu}{2^{j-1}}\right) \mathrm{P}_{\nu}(x) \mathrm{P}_{\nu}(y), \quad j \geq 1, \\
& \Psi_{j}(x, y):=\sum_{\nu=0}^{\infty} \widehat{b}\left(\frac{\nu}{2^{j-1}}\right) \mathrm{P}_{\nu}(x) \mathrm{P}_{\nu}(y), \quad j \geq 1 .
\end{aligned}
$$

Let $\mathcal{X}_{j}$ be the set of knots of the quadrature formula (2.14), defined in (2.13), and let $c_{\xi}$ be the coefficients of the same quadrature. We define the $j$ th level needlets by

$$
\varphi_{\xi}(x):=\mathrm{c}_{\xi}^{1 / 2} \Phi_{j}(x, \xi) \quad \text { and } \quad \psi_{\xi}(x):=\mathrm{c}_{\xi}^{1 / 2} \Psi_{j}(x, \xi), \quad \xi \in \mathcal{X}_{j} .
$$

As in (2.13) we write $\mathcal{X}:=\bigcup_{j=0}^{\infty} \mathcal{X}_{j}$, where equal points from different levels $\mathcal{X}_{j}$ are considered as distinct elements of $\mathcal{X}$, so that $\mathcal{X}$ can be used as an index set. We define the analysis and synthesis needlet systems $\Phi$ and $\Psi$ by

$$
\Phi:=\left\{\varphi_{\xi}\right\}_{\xi \in \mathcal{X}}, \quad \Psi:=\left\{\psi_{\xi}\right\}_{\xi \in \mathcal{X}} .
$$

By estimate (1.10) it follows that the needlets have nearly exponential localization, namely, for $x \in[-1,1]$,

$$
\left|\Phi_{j}(\xi, x)\right|,\left|\Psi_{j}(\xi, x)\right| \leq \frac{c_{\sigma} 2^{j}}{\sqrt{\mathcal{W}\left(2^{j} ; \xi\right)} \sqrt{\mathcal{W}\left(2^{j} ; x\right)}\left(1+2^{j} d(\xi, x)\right)^{\sigma}} \quad \forall \sigma
$$

and hence

$$
\left|\varphi_{\xi}(x)\right|,\left|\psi_{\xi}(x)\right| \leq \frac{c_{\sigma} 2^{j / 2}}{\sqrt{\mathcal{W}\left(2^{j} ; x\right)}\left(1+2^{j} d(\xi, x)\right)^{\sigma}} \quad \forall \sigma .
$$

Note that $x$ in the term $\sqrt{\mathcal{W}\left(2^{j} ; x\right)}$ above can be replaced by $\xi$ (upon replacing $c_{\sigma}$ by a larger constant), namely,

$$
\left|\varphi_{\xi}(x)\right|,\left|\psi_{\xi}(x)\right| \leq \frac{c_{\sigma} 2^{j / 2}}{\sqrt{\mathcal{W}\left(2^{j} ; \xi\right)}\left(1+2^{j} d(\xi, x)\right)^{\sigma}} \quad \forall \sigma .
$$

This estimate follows from (3.10) and (2.22).

We will need to estimate the norms of the needlets. We have, for $0<$ $p \leq \infty$,

$$
\left\|\varphi_{\xi}\right\|_{p} \sim\left\|\psi_{\xi}\right\|_{p} \sim\left\|\widetilde{\mathbb{1}}_{I_{\xi}}\right\|_{p} \sim\left(\frac{2^{j}}{\mathcal{W}\left(2^{j} ; \xi\right)}\right)^{1 / 2-1 / p}, \quad \xi \in \mathcal{X}_{j} .
$$

Moreover, there exist constants $c^{*}, c^{\diamond}>0$ such that

$$
\left\|\varphi_{\xi}\right\|_{L^{\infty}\left(B_{\xi}\left(c^{*} 2^{-j}\right)\right)},\left\|\psi_{\xi}\right\|_{L^{\infty}\left(B_{\xi}\left(c^{*} 2^{-j}\right)\right)} \geq c^{\diamond}\left(\frac{2^{j}}{\mathcal{W}\left(2^{j} ; \xi\right)}\right)^{1 / 2}
$$


where $B_{\xi}\left(c^{*} 2^{-j}\right):=\left\{x \in[-1,1]: d(\xi, x) \leq c^{*} 2^{-j}\right\}$, which is an interval. Notice that if $\widehat{a}, \widehat{b}$ in (3.1)-(3.3) are real-valued then by Proposition 2.4,

$$
\varphi_{\xi}(\xi), \psi_{\xi}(\xi) \geq c\left(\frac{2^{j}}{\mathcal{W}\left(2^{j} ; \xi\right)}\right)^{1 / 2}, \quad c>0 .
$$

For the proofs of (3.12)-(3.13), see the appendix.

Our next goal is to establish needlet decompositions of $\mathcal{D}^{\prime}$ and $L^{p}(w)$.

Proposition 3.1. (a) For $f \in \mathcal{D}^{\prime}$, we have

$$
\begin{aligned}
& f=\sum_{j=0}^{\infty} \Psi_{j} * \bar{\Phi}_{j} * f \quad \text { in } \mathcal{D}^{\prime}, \\
& f=\sum_{\xi \in \mathcal{X}}\left\langle f, \varphi_{\xi}\right\rangle \psi_{\xi} \quad \text { in } \mathcal{D}^{\prime} .
\end{aligned}
$$

(b) If $f \in L^{p}(w), 1 \leq p \leq \infty$, then (3.15)-(3.16) hold in $L^{p}(w)$. Moreover, if $1<p<\infty$, then the convergence in (3.15)-(3.16) is unconditional.

Proof. (a) Let $f \in \mathcal{D}^{\prime}$. By (2.32) and Lemma 2.9, we have

$$
\bar{\Phi}_{j} * f=\sum_{\nu=0}^{2^{j}} \overline{\widehat{a}} \overline{\left(\frac{\nu}{2^{j-1}}\right)} a_{\nu}(f) \mathrm{P}_{\nu}
$$

and further

$$
\Psi_{j} * \bar{\Phi}_{j} * f=\sum_{\nu=0}^{2^{j}} \overline{\widehat{a}\left(\frac{\nu}{2^{j-1}}\right)} \widehat{b}\left(\frac{\nu}{2^{j-1}}\right) a_{\nu}(f) \mathrm{P}_{\nu} .
$$

Now (3.15) follows from (3.4) and Lemmas 2.8-2.9.

Note that $\Psi_{j}(x, y)$ and $\Phi_{j}(x, y)$ are symmetric functions (e.g. $\Psi_{j}(y, x)=$ $\left.\Psi_{j}(x, y)\right)$ and hence $\Psi_{j} * \bar{\Phi}_{j}(x, y)$ is well defined. Also, $\Psi_{j} *\left(\bar{\Phi}_{j} * f\right)=$ $\left(\Psi_{j} * \bar{\Phi}_{j}\right) * f$. We observe that $\Psi_{j}(x, u) \overline{\Phi_{j}(y, u)}$ belongs to $\Pi_{2^{j+1}-1}$ as a function of $u$ and apply the quadrature formula from (2.14) to obtain

$$
\begin{aligned}
\Psi_{j} * \bar{\Phi}_{j}(x, y) & =\int_{-1}^{1} \Psi_{j}(x, u) \overline{\Phi_{j}(y, u)} w(u) d u \\
& =\sum_{\xi \in \mathcal{X}_{j}} \mathrm{c}_{\xi} \Psi_{j}(x, \xi) \overline{\Phi_{j}(y, \xi)}=\sum_{\xi \in \mathcal{X}_{j}} \psi_{\xi}(x) \overline{\varphi_{\xi}(y)} .
\end{aligned}
$$

Hence,

$$
\Psi_{j} * \bar{\Phi}_{j} * f=\sum_{\xi \in \mathcal{X}_{j}}\left\langle f, \varphi_{\xi}\right\rangle \psi_{\xi} .
$$

Substituting this in (3.15) yields (3.16).

(b) To prove (3.15) in $L^{p}(w)$ we observe that $\sum_{j=0}^{l} \Psi_{j} * \bar{\Phi}_{j} * f=L_{l} * f$ with $L_{l}:=\sum_{j=0}^{l} \Psi_{j} * \bar{\Phi}_{j}$. Because of $(3.4), L_{l}(x, y)$ is a reproducing kernel 
for polynomials exactly as the kernels $L_{n}(x, y)$ from Lemma 2.5. Hence, $\sum_{j=0}^{l} \Psi_{j} * \bar{\Phi}_{j} * f \rightarrow f$ in $L^{p}(w)(1 \leq p \leq \infty)$. Then (3.16) in $L^{p}(w)$ follows as above. The unconditional convergence in $L^{p}(w), 1<p<\infty$, follows from Proposition 4.11 and Theorem 4.5 below.

REMARK 3.2. It is easy to see that there exists a function $\widehat{a} \geq 0$ satisfying (3.1)-(3.2) such that $\widehat{a}^{2}(t)+\widehat{a}^{2}(2 t)=1, t \in[1 / 2,1]$. Suppose that in the above construction $\widehat{b}=\widehat{a}$ and $\widehat{a} \geq 0$. Then $\Phi_{j}=\Psi_{j}$ and $\varphi_{\xi}=\psi_{\xi}$. Now (3.16) becomes $f=\sum_{\xi \in \mathcal{X}}\left\langle f, \psi_{\xi}\right\rangle \psi_{\xi}$. It is easily seen that $\left\{\psi_{\xi}: \xi \in \mathcal{X}\right\}$ is a tight frame for $L^{2}(w)$ (see [13]).

4. First scale of weighted Triebel-Lizorkin spaces on $[-1,1]$. In analogy to the classical case on $\mathbb{R}^{d}$ we will define our first scale of weighted Triebel-Lizorkin spaces by means of the Littlewood-Paley expressions employing the kernels $\Phi_{j}$, defined by

$$
\begin{aligned}
& \Phi_{0}(x, y):=\mathrm{P}_{0}(x) \mathrm{P}_{0}(y), \\
& \Phi_{j}(x, y):=\sum_{\nu=0}^{\infty} \widehat{a}\left(\frac{\nu}{2^{j-1}}\right) \mathrm{P}_{\nu}(x) \mathrm{P}_{\nu}(y), \quad j \geq 1,
\end{aligned}
$$

where $\widehat{a}$ satisfies the conditions

$$
\begin{aligned}
& \widehat{a} \in C^{\infty}[0, \infty), \quad \operatorname{supp} \widehat{a} \subset[1 / 2,2], \\
& |\widehat{a}(t)|>c>0 \quad \text { if } t \in[3 / 5,5 / 3] .
\end{aligned}
$$

Definition 4.1. Let $s \in \mathbb{R}, 0<p<\infty$, and $0<q \leq \infty$. Then the weighted Triebel-Lizorkin space $F_{p}^{s q}:=F_{p}^{s q}(w)$ is defined as the set of all $f \in \mathcal{D}^{\prime}$ such that

$$
\|f\|_{F_{p}^{s q}}:=\left\|\left(\sum_{j=0}^{\infty}\left(2^{s j}\left|\Phi_{j} * f(\cdot)\right|\right)^{q}\right)^{1 / q}\right\|_{p}<\infty
$$

with the usual modification when $q=\infty$.

Observe that the above definition is independent of the choice of $\widehat{a}$ as long as it satisfies (4.2)-(4.3) (see Theorem 4.5 below).

Proposition 4.2. For every $s \in \mathbb{R}, 0<p<\infty$, and $0<q \leq \infty, F_{p}^{s q}$ is a quasi-Banach space which is continuously embedded in $\mathcal{D}^{\prime}$.

Proof. We will only prove the continuous embedding of $F_{p}^{s q}$ in $\mathcal{D}^{\prime}$. Then the completeness follows by a standard argument (see e.g. [19, p. 49]).

Suppose the kernels $\Phi_{j}$ are as in the definition of $F_{p}^{s q}$ with $\widehat{a}$ satisfying (4.2)-(4.3) which are the same as (3.1)-(3.2). Then as was already mentioned, there is a function $\widehat{b}$ satisfying (3.1)-(3.2) such that (3.3) holds as well. Let $\Psi_{j}$ be defined by (3.6). Then by Proposition 3.1 any function 
$f \in F_{p}^{s q}$ has the representation $f=\sum_{j=0}^{\infty} \Psi_{j} * \bar{\Phi}_{j} * f$ in $\mathcal{D}^{\prime}$. Hence for $\phi \in \mathcal{D}$ we have $\langle f, \phi\rangle=\sum_{j=0}^{\infty}\left\langle\Psi_{j} * \bar{\Phi}_{j} * f, \phi\right\rangle$. Using (3.17)-(3.18) we find that

$$
\left\langle\Psi_{j} * \bar{\Phi}_{j} * f, \phi\right\rangle=\sum_{\nu=2^{j-2}+1}^{2^{j}} \overline{\widehat{a}\left(\frac{\nu}{2^{j-1}}\right)} \widehat{b}\left(\frac{\nu}{2^{j-1}}\right) a_{\nu}(f) a_{\nu}(\phi) \quad(j \geq 2)
$$

and applying the Cauchy-Schwarz inequality yields

$$
\begin{aligned}
\left|\left\langle\Psi_{j} * \bar{\Phi}_{j} * f, \phi\right\rangle\right| & \leq c 2^{j / 2}\left\|\Phi_{j} * f\right\|_{2} \max _{2^{j-2}<\nu \leq 2^{j}}\left|a_{\nu}(\phi)\right| \\
& \leq c 2^{j(2 / p+1 / 2)}\left\|\Phi_{j} * f\right\|_{p} \max _{2^{j-2}<\nu \leq 2^{j}}\left|a_{\nu}(\phi)\right| \\
& \leq c 2^{-j}\|f\|_{F_{p}^{s q}} \mathcal{N}_{k}(\phi),
\end{aligned}
$$

where $k \geq 2 / p+3 / 2-s, \mathcal{N}_{k}(\cdot)$ is from (2.28), and we used inequality (2.10). Consequently, $|\langle f, \phi\rangle| \leq c\|f\|_{F_{p}^{s q}} \mathcal{N}_{k}(\phi)$, which is the claimed embedding.

Associated to $F_{p}^{s q}$ is the sequence space $f_{p}^{s q}$ defined as follows.

Definition 4.3. Let $s \in \mathbb{R}, 0<p<\infty$, and $0<q \leq \infty$. Then $f_{p}^{s q}$ is defined as the space of all complex-valued sequences $h:=\left\{h_{\xi}\right\}_{\xi \in \mathcal{X}}$ such that

$$
\|h\|_{f_{p}^{s q}}:=\left\|\left(\sum_{j=0}^{\infty} 2^{s j q} \sum_{\xi \in \mathcal{X}_{j}}\left(\left|h_{\xi}\right| \widetilde{\mathbb{1}}_{I_{\xi}}(\cdot)\right)^{q}\right)^{1 / q}\right\|_{p}<\infty
$$

with the usual modification for $q=\infty$. Recall that $\widetilde{\mathbb{1}}_{I_{\xi}}:=\mu\left(I_{\xi}\right)^{-1 / 2} \mathbb{1}_{I_{\xi}}$.

We now introduce the "analysis" and "synthesis" operators

$$
S_{\varphi}: f \mapsto\left\{\left\langle f, \varphi_{\xi}\right\rangle\right\}_{\xi \in \mathcal{X}} \quad \text { and } \quad T_{\psi}:\left\{h_{\xi}\right\}_{\xi \in \mathcal{X}} \mapsto \sum_{\xi \in \mathcal{X}} h_{\xi} \psi_{\xi}
$$

The next lemma shows that the operator $T_{\psi}$ is well defined on $f_{p}^{s q}$.

Lemma 4.4. Let $s \in \mathbb{R}, 0<p<\infty$, and $0<q \leq \infty$. Then for any $h \in f_{p}^{s q}, T_{\psi} h:=\sum_{\xi \in \mathcal{X}} h_{\xi} \psi_{\xi}$ converges in $\mathcal{D}^{\prime}$. Moreover, the operator $T_{\psi}$ : $f_{p}^{s q} \rightarrow \mathcal{D}^{\prime}$ is continuous, that is,

$$
\left|\left\langle T_{\psi} h, \phi\right\rangle\right| \leq c \mathcal{N}_{k}(\phi)\|h\|_{f_{p}^{s q}} \quad \text { for all } h \in f_{p}^{s q}, \phi \in \mathcal{D} .
$$

Proof. Let $h \in f_{p}^{s q}$. Then by the definition of $f_{p}^{s q}$ it follows that

$$
2^{j s}\left|h_{\xi}\right|\left\|\widetilde{\mathbb{1}}_{I_{\xi}}\right\|_{p} \leq\|h\|_{f_{p}^{s q}}, \quad \xi \in \mathcal{X}_{j}, j \geq 0 .
$$

By $(2.19), \mu\left(I_{\xi}\right) \sim 2^{-j} \mathcal{W}\left(2^{j} ; \xi\right)$ and obviously $2^{-(2 \alpha+2 \beta+1) j} \leq \mathcal{W}\left(2^{j} ; \xi\right)$ $\leq 2^{2 \alpha+2 \beta+1}$, which implies

$$
\left\|\widetilde{\mathbb{1}}_{I_{\xi}}\right\|_{p}^{-1}=\mu\left(I_{\xi}\right)^{1 / 2-1 / p} \leq c 2^{j(2 \alpha+2 \beta)|1 / 2-1 / p|} .
$$

Combining this with (4.9) we get

$$
\left|h_{\xi}\right| \leq c 2^{j \gamma_{1}}\|h\|_{f_{p}^{s q}}, \quad \xi \in \mathcal{X}_{j}, \gamma_{1}:=(2 \alpha+2 \beta)|1 / 2-1 / p|-s .
$$


On the other hand, for a given $\phi \in \mathcal{D}$, by Lemma $2.8, \phi=\sum_{n=0}^{\infty}\left\langle\phi, \mathrm{P}_{n}\right\rangle \mathrm{P}_{n}$ in $\mathcal{D}$, and using the definition of $\psi_{\xi}$ in (3.6)-(3.7) we have

$$
\left\langle\psi_{\xi}, \phi\right\rangle=c_{\xi}^{1 / 2} \sum_{\nu=2^{j-2}+1}^{2^{j}} \widehat{b}\left(\frac{\nu}{2^{j-1}}\right)\left\langle\mathrm{P}_{\nu}, \phi\right\rangle \mathrm{P}_{\nu}(\xi) \quad(j \geq 2) .
$$

We use this and the rough estimates $\left\|\mathrm{P}_{\nu}\right\|_{\infty} \leq c \nu^{\alpha+\beta+1 / 2}$ and $c_{\xi} \leq c$ to obtain

$$
\left|\left\langle\psi_{\xi}, \phi\right\rangle\right| \leq c 2^{j \gamma_{2}} \sum_{\nu=2^{j-2}+1}^{2^{j}}\left|\left\langle\mathrm{P}_{\nu}, \phi\right\rangle\right|, \quad \gamma_{2}:=\alpha+\beta+1 / 2 .
$$

Combining this with (4.10) we get

$$
\begin{aligned}
\sum_{\xi \in \mathcal{X}}\left|h_{\xi}\right|\left|\left\langle\psi_{\xi}, \phi\right\rangle\right| & \leq \sum_{j=0}^{\infty} \sum_{\xi \in \mathcal{X}_{j}}\left|h_{\xi}\right|\left|\left\langle\psi_{\xi}, \phi\right\rangle\right| \\
& \leq c\|h\|_{f_{p}^{s q}} \sum_{j=1}^{\infty} 2^{j\left(\gamma_{1}+\gamma_{2}\right)}\left(\# \mathcal{X}_{j}\right) \sum_{2^{j-2} \leq \nu \leq 2^{j}}\left|\left\langle\mathrm{P}_{\nu}, \phi\right\rangle\right| \\
& \leq c\|h\|_{f_{p}^{s q}} \sum_{j=1}^{\infty} 2^{-2 j} \sum_{2^{j-2} \leq \nu \leq 2^{j}}(\nu+1)^{\gamma_{1}+\gamma_{2}+3}\left|\left\langle\mathrm{P}_{\nu}, \phi\right\rangle\right| \\
& \leq c\|h\|_{f_{p}^{s q}} \mathcal{N}_{k}(\phi) \sum_{j=1}^{\infty} 2^{-j} \leq c\|h\|_{f_{p}^{s q}} \mathcal{N}_{k}(\phi)<\infty,
\end{aligned}
$$

where $k:=\left[\gamma_{1}+\gamma_{2}\right]+4$ and for convenience $\mathbf{P}_{1 / 2}:=\mathbf{P}_{0}$. Therefore, the above series converges and hence the series $\sum_{\xi \in \mathcal{X}} h_{\xi} \psi_{\xi}$ converges in $\mathcal{D}^{\prime}$. We define $T_{\psi} h$ by $\left\langle T_{\psi} h, \phi\right\rangle:=\sum_{\xi \in \mathcal{X}} h_{\xi}\left\langle\psi_{\xi}, \phi\right\rangle$ for all $\phi \in \mathcal{D}$. We finally note that estimate (4.8) is immediate from (4.11).

Here is our main result concerning the weighted F-spaces.

Theorem 4.5. Let $s \in \mathbb{R}, 0<p<\infty$ and $0<q \leq \infty$. Then the operators $S_{\varphi}: F_{p}^{s q} \rightarrow f_{p}^{s q}$ and $T_{\psi}: f_{p}^{s q} \rightarrow F_{p}^{s q}$ are bounded and $T_{\psi} \circ S_{\varphi}$ $=\mathrm{Id}$ on $F_{p}^{s q}$. Consequently, for $f \in \mathcal{D}^{\prime}$ we have $f \in F_{p}^{s q}$ if and only if $\left\{\left\langle f, \varphi_{\xi}\right\rangle\right\}_{\xi \in \mathcal{X}} \in f_{p}^{s q}$. Furthermore,

$$
\|f\|_{F_{p}^{s q}} \sim\left\|\left\{\left\langle f, \varphi_{\xi}\right\rangle\right\}\right\|_{f_{p}^{s q}} \sim\left\|\left(\sum_{j=0}^{\infty} 2^{s j q} \sum_{\xi \in \mathcal{X}_{j}}\left|\left\langle f, \varphi_{\xi}\right\rangle \psi_{\xi}(\cdot)\right|^{q}\right)^{1 / q}\right\|_{p} .
$$

In addition, the definition of $F_{p}^{s q}$ is independent of the selection of $\widehat{a}$ satisfying (4.2)-(4.3).

For the proof of this theorem we will need several lemmas whose proofs are given in the appendix. 
Lemma 4.6. If $\xi \in \mathcal{X}_{j}, j \geq 0$, and $0<t<\infty$, then

$$
\begin{gathered}
\left|\varphi_{\xi}(x)\right|,\left|\psi_{\xi}(x)\right| \leq c\left(\mathcal{M}_{t} \widetilde{\mathbb{1}}_{I_{\xi}}\right)(x), \quad x \in[-1,1], \\
\widetilde{\mathbb{1}}_{I_{\xi}}(x) \leq c\left(\mathcal{M}_{t} \varphi_{\xi}\right)(x), c\left(\mathcal{M}_{t} \psi_{\xi}\right)(x), \quad x \in[-1,1] .
\end{gathered}
$$

Lemma 4.7. For any $\sigma>0$ there exists a constant $c_{\sigma}>0$ such that

$$
\begin{aligned}
\left|\Phi_{j} * \psi_{\xi}(x)\right| \leq c_{\sigma} \frac{2^{j / 2}}{\sqrt{\mathcal{W}\left(2^{j} ; x\right)}\left(1+2^{j} d(\xi, x)\right)^{\sigma}}, \\
\quad \xi \in \mathcal{X}_{\nu}, j-1 \leq \nu \leq j+1,
\end{aligned}
$$

and $\Phi_{j} * \psi_{\xi}(x)=0$ for $\xi \in \mathcal{X}_{\nu}, \nu \geq j+2$ or $\nu \leq j-2$. Here $\mathcal{X}_{\nu}:=\emptyset$ if $\nu<0$.

Definition 4.8. For a collection of complex numbers $\left\{h_{\xi}\right\}_{\xi \in \mathcal{X}_{j}}$ we let

$$
h_{\xi}^{*}:=\sum_{\eta \in \mathcal{X}_{j}} \frac{\left|h_{\eta}\right|}{\left(1+2^{j} d(\eta, \xi)\right)^{\sigma}} .
$$

Here $\sigma>1$ is sufficiently large and will be selected later on.

Lemma 4.9. Suppose that $P \in \Pi_{2^{j}}, j \geq 0$, and let $a_{\xi}:=\max _{x \in I_{\xi}}|P(x)|$. There exists $r \geq 1$, depending only on $\sigma, \alpha$, and $\beta$, such that if

$$
b_{\xi}:=\max \left\{\min _{x \in I_{\eta}}|P(x)|: \eta \in \mathcal{X}_{j+r}, I_{\xi} \cap I_{\eta} \neq \emptyset\right\},
$$

then

$$
a_{\xi}^{*} \sim b_{\xi}^{*}
$$

with constants of equivalence independent of $P, j$ and $\xi$.

Lemma 4.10. Assume $t>0$ and let $\left\{b_{\xi}\right\}_{\xi \in \mathcal{X}_{j}}(j \geq 0)$ be a collection of complex numbers. Suppose that $\sigma>(4 \max \{\alpha, \beta\}+3) / t+1$ in the definition (4.16) of $b_{\xi}^{*}$. Then

$$
b_{\xi}^{*} \mathbb{1}_{I_{\xi}}(x) \leq c \mathcal{M}_{t}\left(\sum_{\eta \in \mathcal{X}_{j}}\left|b_{\eta}\right| \mathbb{1}_{I_{\eta}}\right)(x), \quad x \in I_{\xi}, \xi \in \mathcal{X}_{j} .
$$

Proof of Theorem 4.5. Suppose $\alpha \geq \beta$. Fix $0<t<\min \{p, q\}$ and let $\sigma>(4 \alpha+3) / t+1$. We first note that the right-hand side equivalence in (4.12) follows immediately from Lemma 4.6 and the maximal inequality (2.24).

Assume that $\left\{\Phi_{j}\right\}$ are from the definition of weighted Triebel-Lizorkin spaces, i.e. $\Phi_{j}$ are defined by (4.1), where $\widehat{a}$ satisfies (4.2)-(4.3), the same as (3.1)-(3.2). As already mentioned, there exists a function $\widehat{b}$ satisfying (3.1)-(3.2) such that (3.3) holds. Let $\Psi_{j}$ be defined by (3.6) using this $\widehat{b}$. Also, let $\left\{\varphi_{\xi}\right\}_{\xi \in \mathcal{X}}$ and $\left\{\psi_{\xi}\right\}_{\xi \in \mathcal{X}}$ be the associated needlet systems defined as in (3.7).

Further, let $\left\{\widetilde{\Phi}_{j}\right\}$ be a second sequence of kernels like the kernels $\left\{\Phi_{j}\right\}$ above but defined by a different function $\widehat{a}$. Also, we assume that a se- 
quence of companion kernels $\left\{\widetilde{\Psi}_{j}\right\}$ is constructed as above and let $\left\{\widetilde{\varphi}_{\xi}\right\}$, $\left\{\widetilde{\psi}_{\xi}\right\}$ be the associated needlet systems, defined as in (3.5)-(3.7). So, we have two totally different systems of kernels and associated needlet systems.

We first establish the boundedness of $T_{\widetilde{\psi}}: f_{p}^{s q} \rightarrow F_{p}^{s q}$, where the space $F_{p}^{s q}$ is defined by $\left\{\Phi_{j}\right\}$. Let $h \in f_{p}^{s q}$ and define $f:=\sum_{\xi} h_{\xi} \psi_{\xi}$. Using (4.15) we have, for $x \in[-1,1]$,

$$
\begin{aligned}
\left|\Phi_{j} * f(x)\right| & =\left|\sum_{\xi \in \mathcal{X}} h_{\xi} \Phi_{j} * \widetilde{\psi}_{\xi}(x)\right| \leq \sum_{j-1 \leq \nu \leq j+1} \sum_{\xi \in \mathcal{X}_{\mu}}\left|h_{\xi}\right|\left|\Phi_{j} * \widetilde{\psi}_{\xi}(x)\right| \\
& \leq c 2^{j / 2} \sum_{j-1 \leq \nu \leq j+1} \sum_{\xi \in \mathcal{X}_{\nu}} \frac{\left|h_{\xi}\right|}{\sqrt{\mathcal{W}\left(2^{\nu} ; x\right)}\left(1+2^{\nu} d(\xi, x)\right)^{\sigma}} .
\end{aligned}
$$

Fix $\eta \in \mathcal{X}_{j}$ and set $\mathcal{Y}_{\eta}:=\left\{\xi \in \mathcal{X}_{j-1} \cup \mathcal{X}_{j} \cup \mathcal{X}_{j+1}: I_{\xi} \cap I_{\eta} \neq \emptyset\right\}\left(\mathcal{X}_{-1}:=\emptyset\right)$. Notice that $\# \mathcal{Y}_{\eta} \leq$ const and $d(x, \xi) \leq c 2^{-j}$ if $x \in I_{\xi}$ and $\xi \in \mathcal{Y}_{\eta}$. Hence, we have, for $x \in I_{\eta}$,

$$
\begin{aligned}
\left|\Phi_{j} * f(x)\right| & \leq c 2^{j / 2} \sum_{j-1 \leq \nu \leq j+1} \sum_{\omega \in \mathcal{Y}_{\eta} \cap \mathcal{X}_{\nu}} \sum_{\xi \in \mathcal{X}_{\nu}} \frac{\left|h_{\xi}\right| \mathbb{1}_{\omega}(x)}{\sqrt{\mathcal{W}\left(2^{\nu} ; \omega\right)}\left(1+2^{\nu} d(\xi, \omega)\right)^{\sigma}} \\
& \leq c 2^{j / 2} \sum_{\omega \in \mathcal{Y}_{\eta}} \frac{h_{\omega}^{*} \mathbb{1}_{\omega}(x)}{\sqrt{\mathcal{W}\left(2^{j} ; \omega\right)}} \leq c \sum_{\omega \in \mathcal{Y}_{\eta}} h_{\omega}^{*} \widetilde{\mathbb{1}}_{\omega}(x),
\end{aligned}
$$

where we also used (2.19). We now insert this in (4.4) and use Lemma 4.10 and the maximal inequality (2.24) to obtain

$$
\begin{aligned}
\|f\|_{F_{p}^{s q}} & \leq c\left\|\left(\sum_{j=0}^{\infty}\left[2^{s j} \sum_{\eta \in \mathcal{X}_{j}} \sum_{\omega \in \mathcal{Y}_{\eta}} h_{\omega}^{*} \widetilde{\mathbb{1}}_{I_{\omega}}(\cdot)\right]^{q}\right)^{1 / q}\right\|_{p} \\
& \leq c\left\|\left(\sum_{j=0}^{\infty}\left[2^{s j} \sum_{\xi \in \mathcal{X}_{j}} h_{\xi}^{*} \widetilde{\mathbb{1}}_{I_{\xi}}(\cdot)\right]^{q}\right)^{1 / q}\right\|_{p} \\
& \leq c\left\|\left(\sum_{j=0}^{\infty}\left[\mathcal{M}_{t}\left(\sum_{\xi \in \mathcal{X}_{j}} 2^{s j}\left|h_{\xi}\right| \widetilde{\mathbb{1}}_{I_{\xi}}\right)(\cdot)\right]^{q}\right)^{1 / q}\right\|_{p} \leq c\left\|\left\{h_{\xi}\right\}\right\|_{f_{p}^{s q}}
\end{aligned}
$$

For the second estimate above it was important that $\# \mathcal{Y}_{\eta} \leq c$. Therefore, the operator $T_{\widetilde{\psi}}: f_{p}^{s q} \rightarrow F_{p}^{s q}$ is bounded.

We next prove the boundedness of the operator $S_{\varphi}: F_{p}^{s q} \rightarrow f_{p}^{s q}$, where we assume this time that $F_{p}^{s q}$ is defined in terms of $\left\{\bar{\Phi}_{j}\right\}$. Let $f \in F_{p}^{s q}$. Then $\bar{\Phi}_{j} * f \in \Pi_{2^{j}}$. For $\xi \in \mathcal{X}_{j}$, we set $a_{\xi}:=\max _{x \in I_{\xi}}\left|\bar{\Phi}_{j} * f(x)\right|, \quad b_{\xi}:=\max \left\{\min _{x \in I_{\eta}}\left|\bar{\Phi}_{j} * f(x)\right|: \eta \in \mathcal{X}_{j+r}, I_{\xi} \cap I_{\eta} \neq \emptyset\right\}$. 
Assuming that $r$ above is the constant from Lemma 4.9, it follows from the same lemma that $a_{\xi}^{*} \sim b_{\xi}^{*}$. Therefore,

$$
\left|\left\langle f, \varphi_{\xi}\right\rangle\right|=\mathrm{c}_{\xi}^{1 / 2}\left|\bar{\Phi}_{j} * f(\xi)\right| \leq c \mu\left(I_{\xi}\right)^{1 / 2} a_{\xi} \leq c \mu\left(I_{\xi}\right)^{1 / 2} a_{\xi}^{*} \leq c \mu\left(I_{\xi}\right)^{1 / 2} b_{\xi}^{*} .
$$

From this, taking into account that $\widetilde{\mathbb{1}}_{I_{\xi}}:=\mu\left(I_{\xi}\right)^{-1 / 2} \mathbb{1}_{I_{\xi}}$, we obtain

$$
\begin{aligned}
\left\|\left\{\left\langle f, \varphi_{\xi}\right\rangle\right\}\right\|_{f_{p}^{s q}} & :=\left\|\left(\sum_{j \geq 0} 2^{j s q} \sum_{\xi \in \mathcal{X}_{j}}\left[\left|\left\langle f, \varphi_{\xi}\right\rangle\right| \widetilde{\mathbb{1}}_{I_{\xi}}(\cdot)\right]^{q}\right)^{1 / q}\right\|_{p} \\
& \leq c\left\|\left(\sum_{j \geq 0} 2^{j s q} \sum_{\xi \in \mathcal{X}_{j}}\left[b_{\xi}^{*} \mathbb{1}_{I_{\xi}}(\cdot)\right]^{q}\right)^{1 / q}\right\|_{p} \\
& \leq c\left\|\left(\sum_{j \geq 0} 2^{j s q}\left[\mathcal{M}_{t}\left(\sum_{\xi \in \mathcal{X}_{j}} b_{\xi} \mathbb{1}_{I_{\xi}}\right)(\cdot)\right]^{q}\right)^{1 / q}\right\|_{p} \\
& \leq c\left\|\left(\sum_{j \geq 0} 2^{j s q} \sum_{\xi \in \mathcal{X}_{j}} b_{\xi}^{q} \mathbb{1}_{I_{\xi}}(\cdot)\right)^{1 / q}\right\|_{p}
\end{aligned}
$$

where for the second inequality above we used Lemma 4.10 and for the third the maximal inequality $(2.24)$.

Let $m_{\eta}:=\min _{x \in I_{\eta}}\left|\bar{\Phi}_{j} * f(x)\right|$ for $\xi \in \mathcal{X}_{j+r}$ and define, for $\xi \in \mathcal{X}_{j}$,

$$
\mathcal{X}_{j+r}(\xi):=\left\{w \in \mathcal{X}_{j+r}: I_{w} \cap I_{\xi} \neq \emptyset\right\} .
$$

Evidently, $\# \mathcal{X}_{j+r}(\xi) \leq \widetilde{c}, \widetilde{c}=\widetilde{c}(r)$. Hence, $d(w, \eta) \leq c(r) 2^{-j-r}$ for $w, \eta \in$ $\mathcal{X}_{j+r}(\xi)$ and therefore

$$
m_{w} \leq c \frac{m_{w}}{1+2^{j+r} d(w, \eta)} \leq c m_{\eta}^{*}
$$

Consequently, for any $\xi \in \mathcal{X}_{j}$ and $\eta \in \mathcal{X}_{j+r}(\xi)$, we have $b_{\xi}=\max _{w \in \mathcal{X}_{j+r}(\xi)} m_{w}$ $\leq c m_{\eta}^{*}$ and hence

$$
b_{\xi} \mathbb{1}_{I_{\xi}} \leq c \sum_{\eta \in \mathcal{X}_{j+r}(\xi)} m_{\eta}^{*} \mathbb{1}_{I_{\eta}} .
$$

Using this estimate in (4.19) we get

$$
\begin{aligned}
\left\|\left\{\left\langle f, \varphi_{\xi}\right\rangle\right\}\right\|_{f_{p}^{s q}} & \leq c\left\|\left(\sum_{j \geq 0} 2^{j s q}\left(\sum_{\eta \in \mathcal{X}_{j+r}} m_{\eta}^{*} \mathbb{1}_{I_{\eta}}(\cdot)\right)^{q}\right)^{1 / q}\right\|_{p} \\
& \leq c\left\|\left(\sum_{j \geq 0} 2^{j s q}\left[\mathcal{M}_{t}\left(\sum_{\eta \in \mathcal{X}_{j+r}} m_{\eta} \mathbb{1}_{I_{\eta}}\right)(\cdot)\right]^{q}\right)^{1 / q}\right\|_{p} \\
& \leq c\left\|\left(\sum_{j \geq 0}\left(2^{j s} \sum_{\xi \in \mathcal{X}_{j}} m_{\xi} \mathbb{1}_{I_{\xi}}(\cdot)\right)^{q}\right)^{1 / q}\right\|_{p} \\
& \leq c\left\|\left(\sum_{j \geq 0}\left(2^{j s}\left|\bar{\Phi}_{j} * f\right|\right)^{q}\right)^{1 / q}\right\|_{p} .
\end{aligned}
$$

Thus the boundedness of $S_{\varphi}: F_{p}^{s q} \rightarrow f_{p}^{s q}$ is established. 
The identity $T_{\psi} \circ S_{\varphi}=$ Id follows from Proposition 3.1.

It remains to show that $F_{p}^{s q}$ is independent of the particular selection of $\widehat{a}$ in the definition of $\left\{\Phi_{j}\right\}$. Denote for the moment by $\|f\|_{F_{p}^{s q}(\Phi)}$ the F-norm defined by $\left\{\Phi_{j}\right\}$. Then by the above proof it follows that

$$
\|f\|_{F_{p}^{s q}(\Phi)} \leq c\left\|\left\{\left\langle f, \widetilde{\varphi}_{\xi}\right\rangle\right\}\right\|_{f_{p}^{s q}} \quad \text { and } \quad\left\|\left\{\left\langle f, \varphi_{\xi}\right\rangle\right\}\right\|_{f_{p}^{s q}} \leq c\|f\|_{F_{p}^{s q}(\bar{\Phi})}
$$

and hence

$$
\|f\|_{F_{p}^{s q}(\Phi)} \leq c\left\|\left\{\left\langle f, \widetilde{\varphi}_{\xi}\right\rangle\right\}\right\|_{f_{p}^{s q}} \leq c\|f\|_{F_{p}^{s q}(\overline{\widetilde{\Phi}})} .
$$

Now the desired independence follows by reversing the roles of $\left\{\Phi_{j}\right\},\left\{\widetilde{\Phi}_{j}\right\}$, and their complex conjugates.

It is natural to define the weighted potential space (generalized weighted Sobolev space) $H_{s}^{p}:=H_{s}^{p}(w), s>0,1 \leq p \leq \infty$, on $[-1,1]$ as the set of all $f \in \mathcal{D}^{\prime}$ such that

$$
\|f\|_{H_{s}^{p}}:=\left\|\sum_{n=0}^{\infty}(n+1)^{s} a_{n}(f) \mathrm{P}_{n}(\cdot)\right\|_{p}<\infty,
$$

where $a_{n}(f):=\left\langle f, \mathrm{P}_{n}\right\rangle$ as in Lemma 2.9 .

In the next statement we identify certain weighted Triebel-Lizorkin spaces as weighted potential spaces or $L^{p}(w)$.

Proposition 4.11. We have

$$
F_{p}^{s 2} \sim H_{s}^{p}, \quad s>0,1<p<\infty,
$$

and

$$
F_{p}^{02} \sim H_{0}^{p} \sim L^{p}(w), \quad 1<p<\infty,
$$

with equivalent norms. Therefore, for any $f \in L^{p}(w), 1<p<\infty$,

$$
\|f\|_{p} \sim\left\|\left(\sum_{j=0}^{\infty} \sum_{\xi \in \mathcal{X}_{j}}\left|\left\langle f, \varphi_{\xi}\right\rangle \psi_{\xi}(\cdot)\right|^{2}\right)^{1 / 2}\right\|_{p} .
$$

One proves this proposition in a standard way using e.g. the multipliers from [3]. The proof can be carried out exactly as in the case of spherical harmonic expansions, given in [11, Proposition 4.3], and will be omitted.

5. Second scale of weighted Triebel-Lizorkin spaces on $[-1,1]$. We introduce our second scale of Triebel-Lizorkin spaces by utilizing again the kernels $\Phi_{j}$ defined by (4.1) with $\widehat{a}$ satisfying (4.2)-(4.3) (compare with $\S 4)$.

Definition 5.1. Let $s \in \mathbb{R}, 0<p<\infty$, and $0<q \leq \infty$. Then the weighted Triebel-Lizorkin space $\mathcal{F}_{p}^{s q}:=\mathcal{F}_{p}^{s q}(w)$ is defined as the set of all 
$f \in \mathcal{D}^{\prime}$ such that

$$
\|f\|_{\mathcal{F}_{p}^{s q}}:=\left\|\left(\sum_{j=0}^{\infty}\left[2^{s j} \mathcal{W}\left(2^{j} ; \cdot\right)^{-s}\left|\Phi_{j} * f(\cdot)\right|\right]^{q}\right)^{1 / q}\right\|_{p}<\infty
$$

with the usual modification when $q=\infty$.

Observe that the above definition is independent of the choice of $\widehat{a}$ as long as $\widehat{a}$ satisfies (4.2)-(4.3) (see Theorem 5.3 below). Following in the footsteps of the development from $\S 4$, it is easy to show that $\mathcal{F}_{p}^{s q}$ is a complete quasi-Banach space, which is continuously embedded in $\mathcal{D}^{\prime}$. For the latter one proceeds as in the proof of Proposition 4.2, where in (4.5) one, in addition, uses the obvious estimate $\|g\|_{2} \leq c n^{\gamma}\left\|\mathcal{W}(n ; \cdot)^{s} g(\cdot)\right\|_{2}$, where $\gamma:=(2 \min \{\alpha, \beta\}+1) s_{+}$, which is immediate from $c_{1} n^{-2 \min \{\alpha, \beta\}-1} \leq$ $\mathcal{W}(n ; x) \leq c_{2}, x \in[-1,1]$. We skip the details.

The sequence space $\mathbf{f}_{p}^{s q}$ associated with $\mathcal{F}_{p}^{s q}$ is now defined as follows.

Definition 5.2. Let $s \in \mathbb{R}, 0<p<\infty$, and $0<q \leq \infty$. Then $\mathbf{f}_{p}^{s q}$ is defined as the space of all complex-valued sequences $h:=\left\{h_{\xi}\right\}_{\xi \in \mathcal{X}}$ such that

$$
\|h\|_{\mathbf{f}_{p}^{s q}}:=\left\|\left(\sum_{\xi \in \mathcal{X}}\left[\mu\left(I_{\xi}\right)^{-s} \mid h_{\xi} \widetilde{\mathbb{1}}_{I_{\xi}}(\cdot)\right]^{q}\right)^{1 / q}\right\|_{p}<\infty
$$

with the usual modification when $q=\infty$.

To characterize the Triebel-Lizorkin spaces $\mathcal{F}_{p}^{s q}$ we use again the operators $S_{\varphi}$ and $T_{\psi}$ from (4.7). (One shows that $T_{\psi}$ is well defined on $\mathbf{f}_{p}^{s q}$ in much the same way as in Lemma 4.4.)

Theorem 5.3. Let $s \in \mathbb{R}, 0<p<\infty$ and $0<q \leq \infty$. Then the operators $S_{\varphi}: \mathcal{F}_{p}^{s q} \rightarrow \mathbf{f}_{p}^{s q}$ and $T_{\psi}: \mathbf{f}_{p}^{s q} \rightarrow \mathcal{F}_{p}^{s q}$ are bounded and $T_{\psi} \circ S_{\varphi}=\mathrm{Id}$ on $\mathcal{F}_{p}^{s q}$. Consequently, for $f \in \mathcal{D}^{\prime}$ we have that $f \in \mathcal{F}_{p}^{s q}$ if and only if $\left\{\left\langle f, \varphi_{\xi}\right\rangle\right\}_{\xi \in \mathcal{X}} \in \mathbf{f}_{p}^{s q}$. Furthermore,

$$
\|f\|_{\mathcal{F}_{p}^{s q}} \sim\left\|\left\{\left\langle f, \varphi_{\xi}\right\rangle\right\}\right\|_{\mathbf{f}_{p}^{s q}} \sim\left\|\left(\sum_{\xi \in \mathcal{X}}\left[\mu\left(I_{\xi}\right)^{-s}\left|\left\langle f, \varphi_{\xi}\right\rangle \psi_{\xi}(\cdot)\right|\right]^{q}\right)^{1 / q}\right\|_{p} .
$$

In addition, the definition of $\mathcal{F}_{p}^{s q}$ is independent of the selection of $\widehat{a}$ satisfying (4.2)-(4.3).

The proof of this theorem is similar to the proof of Theorem 4.5. The only new ingredient is the following lemma.

Lemma 5.4. Let $t>0$ and $s \in \mathbb{R}$. Suppose $\left\{b_{\xi}\right\}_{\xi \in \mathcal{X}_{j}}(j \geq 0)$ is a collection of complex numbers and let $\sigma>(4 \max \{\alpha, \beta\}+3)(1 / t+|s|)+1$ in the definition (4.16) of $b_{\xi}^{*}$. Then

$$
\mu\left(I_{\xi}\right)^{-s} b_{\xi}^{*} \mathbb{1}_{I_{\xi}}(x) \leq c \mathcal{M}_{t}\left(\sum_{\eta \in \mathcal{X}_{j}} \mu\left(I_{\eta}\right)^{-s}\left|b_{\eta}\right| \mathbb{1}_{I_{\eta}}\right)(x), \quad x \in I_{\xi}, \xi \in \mathcal{X}_{j},
$$


Proof. For $\xi \in \mathcal{X}_{j}, \mu\left(I_{\xi}\right) \sim 2^{-j} \mathcal{W}\left(2^{j} ; \xi\right)$ and hence, using (2.22),

$$
\begin{aligned}
\mu\left(I_{\xi}\right)^{-s} b_{\xi}^{*} & \leq c \sum_{\eta \in \mathcal{X}_{j}} \frac{2^{j s} \mathcal{W}\left(2^{j} ; \xi\right)^{-s}\left|b_{\eta}\right|}{\left(1+2^{j} d(\xi, \eta)\right)^{\sigma}} \\
& \leq c \sum_{\eta \in \mathcal{X}_{j}} \frac{2^{j s} \mathcal{W}\left(2^{j} ; \eta\right)^{-s}\left|b_{\eta}\right|}{\left(1+2^{j} d(\xi, \eta)\right)^{\sigma_{1}}} \leq c\left(\mu\left(I_{\eta}\right)^{-s}\left|b_{\eta}\right|\right)^{*},
\end{aligned}
$$

where $\sigma_{1}:=\sigma-(2 \max \{\alpha, \beta\}+1)|s|>(4 \max \{\alpha, \beta\}+3) / t+1$. Now (5.4) follows from Lemma 4.10.

Now the proof of Theorem 5.3 can be carried out as the proof of Theorem 4.5, using Lemma 5.4 in place of Lemma 4.10 and selecting $\sigma$ in the definitions of $h_{\xi}^{*}$ and $a_{\xi}^{*}, b_{\xi}^{*}$ sufficiently large. We skip the further details.

In a sense the spaces $\mathcal{F}_{p}^{s q}$ are more natural than the spaces $F_{p}^{s q}$ from $\S 4$ since they embed "correctly" with respect to the smoothness index $s$.

Proposition 5.5. Let $0<p<p_{1}<\infty, 0<q, q_{1} \leq \infty$, and $-\infty<$ $s_{1}<s<\infty$. Then we have the continuous embedding

$$
\mathcal{F}_{p}^{s q} \subset \mathcal{F}_{p_{1}}^{s_{1} q_{1}} \quad \text { if } s-1 / p=s_{1}-1 / p_{1} .
$$

The proof of this embedding result can be carried out as the proof of Proposition 4.11 in [9] (the argument is similar to the one in the classical case of $\mathbb{R}^{n}$, see e.g. [19, p. 129]). We omit it.

6. First scale of weighted Besov spaces on $[-1,1]$. To introduce the first scale of weighted Besov spaces we use the kernels $\Phi_{j}$ defined in (4.1) with $\widehat{a}$ satisfying $(4.2)-(4.3)$ (see $[16,19]$ ).

Definition 6.1. Let $s \in \mathbb{R}$ and $0<p, q \leq \infty$. Then the weighted Besov space $B_{p}^{s q}:=B_{p}^{s q}(w)$ is defined as the set of all $f \in \mathcal{D}^{\prime}$ such that

$$
\|f\|_{B_{p}^{s q}}:=\left(\sum_{j=0}^{\infty}\left(2^{s j}\left\|\Phi_{j} * f\right\|_{p}\right)^{q}\right)^{1 / q}<\infty,
$$

where the $l_{q}$-norm is replaced by the sup-norm if $q=\infty$.

Note that as in the case of weighted Triebel-Lizorkin spaces the above definition is independent of the choice of $\widehat{a}$ satisfying (4.2)-(4.3) (see Theorem 6.4). Also, the Besov space $B_{p}^{s q}(w)$ is a quasi-Banach space which is continuously embedded in $\mathcal{D}^{\prime}$.

It is natural to associate to the weighted Besov space $B_{p}^{s q}$ the sequence space $b_{p}^{s q}$ defined as follows.

Definition 6.2. Let $s \in \mathbb{R}$ and $0<p, q \leq \infty$. Then $b_{p}^{s q}:=b_{p}^{s q}(w)$ is defined to be the space of all complex-valued sequences $h:=\left\{h_{\xi}\right\}_{\xi \in \mathcal{X}}$ such 
that

$$
\|h\|_{b_{p}^{s q}}:=\left(\sum_{j=0}^{\infty} 2^{j s q}\left[\sum_{\xi \in \mathcal{X}_{j}}\left(\mu\left(I_{\xi}\right)^{1 / p-1 / 2}\left|h_{\xi}\right|\right)^{p}\right]^{q / p}\right)^{1 / q}<\infty
$$

with the usual modification for $p=\infty$ or $q=\infty$.

The analysis and synthesis operators $S_{\varphi}$ and $T_{\psi}$ defined in (4.7) will play a distinctive role in this section. The next lemma shows that the operator $T_{\psi}$ is well defined on $b_{p}^{s q}$.

Lemma 6.3. Let $s \in \mathbb{R}, 0<p, q \leq \infty$. Then for any $h \in b_{p}^{s q}, T_{\psi} h:=$ $\sum_{\xi \in \mathcal{X}} h_{\xi} \psi_{\xi}$ converges in $\mathcal{D}^{\prime}$. Moreover, the operator $T_{\psi}: b_{p}^{s q} \rightarrow \mathcal{D}^{\prime}$ is continuous.

The proof of this lemma is similar to the proof of Lemma 4.4 and will be omitted.

Our main result in this section is the following characterization of weighted Besov spaces.

Theorem 6.4. Let $s \in \mathbb{R}$ and $0<p, q \leq \infty$. The operators $S_{\varphi}$ : $B_{p}^{s q} \rightarrow b_{p}^{s q}$ and $T_{\psi}: b_{p}^{s q} \rightarrow B_{p}^{s q}$ are bounded and $T_{\psi} \circ S_{\varphi}=\operatorname{Id}$ on $B_{p}^{s q}$. Consequently, for $f \in \mathcal{D}^{\prime}$ we have $f \in B_{p}^{s q}$ if and only if $\left\{\left\langle f, \varphi_{\xi}\right\rangle\right\}_{\xi \in \mathcal{X}} \in b_{p}^{s q}$. Moreover,

$$
\|f\|_{B_{p}^{s q}} \sim\left\|\left\{\left\langle f, \varphi_{\xi}\right\rangle\right\}\right\|_{b_{p}^{s q}} \sim\left(\sum_{j=0}^{\infty} 2^{s j q}\left[\sum_{\xi \in \mathcal{X}_{j}}\left\|\left\langle f, \varphi_{\xi}\right\rangle \psi_{\xi}\right\|_{p}^{p}\right]^{q / p}\right)^{1 / q} .
$$

In addition, the definition of $B_{p}^{s q}$ is independent of the selection of $\widehat{a}$ satisfying (4.2)-(4.3).

To prove this theorem we will need the following lemma whose proof is presented in the appendix.

Lemma 6.5. For every $P \in \Pi_{2^{j}}, j \geq 0$, and $0<p \leq \infty$,

$$
\left(\sum_{\xi \in \mathcal{X}_{j}} \max _{x \in I_{\xi}}|P(x)|^{p} \mu\left(I_{\xi}\right)\right)^{1 / p} \leq c\|P\|_{p} .
$$

Proof of Theorem 6.4. Note first that the right-hand equivalence of (6.1) follows immediately from (3.12).

As in the proof of Theorem 4.5, assume that the kernels $\Phi_{j}$ are defined by (4.1), where $\widehat{a}$ satisfies (4.2)-(4.3). Let $\widehat{b}$ be such that (3.1)-(3.3) hold and let $\Psi_{j}$ be defined by (3.6) using this $\widehat{b}$. Also, let $\left\{\varphi_{\xi}\right\}_{\xi \in \mathcal{X}}$ and $\left\{\psi_{\xi}\right\}_{\xi \in \mathcal{X}}$ be the associated needlet systems defined as in (3.7). Further, assume that $\left\{\widetilde{\Phi}_{j}\right\},\left\{\widetilde{\Psi}_{j}\right\},\left\{\widetilde{\varphi}_{\xi}\right\},\left\{\widetilde{\psi}_{\xi}\right\}$ is a second set of kernels and needlets.

We first prove the boundedness of the operator $T_{\widetilde{\psi}}: b_{p}^{s q} \rightarrow B_{p}^{s q}$, where $B_{p}^{s q}$ is defined via $\left\{\Phi_{j}\right\}$. Let $0<t<\min \{p, 1\}$ and $\sigma \geq(2 \alpha+2) / t+\alpha+1 / 2$. 
Assume $h \in b_{p}^{s q}$ and set $f:=\sum_{\xi \in \mathcal{X}} h_{\xi} \psi_{\xi}$. Employing Lemmata 2.7, 4.7, and (2.22) we get

$$
\begin{aligned}
\left|\Phi_{j} * f(x)\right| & \leq \sum_{j-1 \leq \nu \leq j+1} \sum_{\xi \in \mathcal{X}_{\nu}}\left|h_{\xi}\right|\left|\Phi_{j} * \widetilde{\psi}_{\xi}(x)\right| \\
& \leq c \sum_{j-1 \leq \nu \leq j+1} \sum_{\xi \in \mathcal{X}_{\nu}}\left|h_{\xi}\right| \frac{2^{j / 2}}{\sqrt{\mathcal{W}\left(2^{j} ; x\right)}\left(1+2^{j} d(\xi, x)\right)^{\sigma}} \\
& \leq c \sum_{j-1 \leq \nu \leq j+1} \sum_{\xi \in \mathcal{X}_{\nu}}\left|h_{\xi}\right| \frac{2^{j / 2}}{\sqrt{\mathcal{W}\left(2^{j} ; \xi\right)}\left(1+2^{j} d(\xi, x)\right)^{\sigma-\alpha-1 / 2}} \\
& \leq c \sum_{j-1 \leq \nu \leq j+1} \sum_{\xi \in \mathcal{X}_{\nu}}\left|h_{\xi}\right| \mu\left(I_{\xi}\right)^{-1 / 2} \mathcal{M}_{t}\left(\mathbb{1}_{I_{\xi}}\right)(x) \quad\left(\mathcal{X}_{-1}:=\emptyset\right),
\end{aligned}
$$

where we also used the inequality $\sigma \geq(2 \alpha+2) / t+\alpha+1 / 2$. Using the maximal inequality (2.24) it follows that

$$
\begin{aligned}
\left\|\Phi_{j} * f\right\|_{p}^{p} & \leq\left\|\sum_{j-1 \leq \nu \leq j+1} \sum_{\xi \in \mathcal{X}_{\nu}}\left|h_{\xi}\right| \mu\left(I_{\xi}\right)^{-1 / 2} \mathcal{M}_{t}\left(\mathbb{1}_{I_{\xi}}\right)(\cdot)\right\|_{p}^{p} \\
& \leq c \sum_{j-1 \leq \nu \leq j+1} \sum_{\xi \in \mathcal{X}_{\nu}}\left|h_{\xi}\right|^{p} \mu\left(I_{\xi}\right)^{-p / 2} \int_{-1}^{1} \mathbb{1}_{I_{\xi}}(x) w(x) d x \\
& \leq c \sum_{j-1 \leq \nu \leq j+1} \sum_{\xi \in \mathcal{X}_{\nu}}\left|h_{\xi}\right|^{p} \mu\left(I_{\xi}\right)^{1-p / 2} .
\end{aligned}
$$

Multiplying by $2^{j s}$ and summing over $j \geq 0$ we get $\|f\|_{B_{p}^{s q}} \leq c\left\|\left\{h_{\xi}\right\}\right\|_{b_{p}^{s q}}$.

We next prove the boundedness of the operator $S_{\varphi}: B_{p}^{s q} \rightarrow b_{p}^{s q}$, where we assume that $B_{p}^{s q}$ is defined in terms of $\left\{\bar{\Phi}_{j}\right\}$. Note first that

$$
\left|\left\langle f, \varphi_{\xi}\right\rangle\right| \sim \mu\left(I_{\xi}\right)^{1 / 2}\left|\bar{\Phi}_{j} * f(\xi)\right|, \quad \xi \in \mathcal{X}_{j} .
$$

Since $\bar{\Phi}_{j} * f \in \Pi_{2^{j}}$, by Lemma 6.5 we obtain

$$
\sum_{\xi \in \mathcal{X}_{j}} \mu\left(I_{\xi}\right)^{1-p / 2}\left|\left\langle f, \varphi_{\xi}\right\rangle\right|^{p} \leq c \sum_{\xi \in \mathcal{X}_{j}} \mu\left(I_{\xi}\right) \sup _{x \in I_{\xi}}\left|\bar{\Phi}_{j} * f(x)\right|^{p} \leq c\left\|\bar{\Phi}_{j} * f\right\|_{p}^{p},
$$

which yields $\|\{\langle f, \varphi\rangle\}\|_{b_{p}^{s q}} \leq c\|f\|_{B_{p}^{s q}}$.

The identity $T_{\psi} \circ S_{\varphi}=$ Id follows from Proposition 3.1.

The independence of $B_{p}^{s q}$ from the particular selection of $\widehat{a}$ in the definition of $\left\{\Phi_{j}\right\}$ follows from the above exactly as in the Triebel-Lizorkin case (see the proof of Theorem 4.5).

Our next goal is to link the weighted Besov spaces with best polynomial approximation in $L^{p}(w)$. Denote by $A_{p}^{s q}$ the approximation space of all 
functions $f \in L^{p}(w)$ such that

$$
\|f\|_{A_{p}^{s q}}:=\|f\|_{p}+\left(\sum_{j=0}^{\infty}\left(2^{s j} E_{2^{j}}(f)_{p}\right)^{q}\right)^{1 / q}<\infty,
$$

where $E_{n}(f)_{p}$ denotes the best approximation of $f \in L^{p}(w)$ from $\Pi_{n}$ (see $(2.7))$.

Proposition 6.6. Let $s>0,1 \leq p \leq \infty$, and $0<q \leq \infty$. Then $f \in B_{p}^{s q}$ if and only if $f \in A_{p}^{s q}$. Moreover,

$$
\|f\|_{A_{p}^{s q}} \sim\|f\|_{B_{p}^{s q}} .
$$

Proof. Let $f \in B_{p}^{s q}$. It is easy and standard to show that under the assumptions on $s, p$, and $q$ the space $B_{p}^{s q}$ is continuously embedded in $L^{p}(w)$, i.e. $f$ can be identified as a function in $L^{p}(w)$ and $\|f\|_{p} \leq c\|f\|_{B_{p}^{s q}}$.

It is easy to construct (see e.g. [6]) a function $\widehat{a} \geq 0$ satisfying (4.2)-(4.3) such that $\widehat{a}(t)+\widehat{a}(2 t)=1$ for $t \in[1 / 2,1]$ and hence

$$
\sum_{\nu=0}^{\infty} \widehat{a}\left(2^{-\nu} t\right)=1, \quad t \in[1, \infty) .
$$

Assume that $\left\{\Phi_{j}\right\}$ are defined by (4.1) with such an $\widehat{a}$. As in Proposition 3.1, it is easy to see that $f=\sum_{j=0}^{\infty} \Phi_{j} * f$ in $L^{p}(w)$. Hence, since $\Phi_{j} * f \in \Pi_{2^{j}}$,

$$
E_{2^{l}}(f)_{p} \leq \sum_{j=l+1}^{\infty}\left\|\Phi_{j} * f\right\|_{p}, \quad l \geq 0 .
$$

Now, a standard argument using (6.6) shows that $\|f\|_{A_{p}^{s q}} \leq c\|f\|_{B_{p}^{s q}}$.

To prove the estimate in the other direction, we note that $\Phi_{j} * f=\Phi_{j} *$ $(f-Q)$ for $Q \in \Pi_{2^{j-2}}(j \geq 2)$. Hence, as in Lemma $2.5,\left\|\Phi_{j} * f\right\|_{p} \leq c\|f-Q\|_{p}$. Therefore,

$$
\left\|\Phi_{j} * f\right\|_{p} \leq c E_{2^{j-2}}(f)_{p}, \quad j \geq 2, \quad\left\|\Phi_{j} * f\right\|_{p} \leq c\|f\|_{p},
$$

which implies $\|f\|_{B_{p}^{s q}} \leq c\|f\|_{A_{p}^{s q}}$.

Above we used the fact that the definition of $B_{p}^{s q}$ is independent of the selection of $\widehat{a}$, satisfying (4.2)-(4.3).

REMARK 6.7. It is worth mentioning that $E_{n}(f)_{p}$ can be characterized via the weighted moduli of smoothness of Ditzian-Totik [4]. Consequently, the weighted moduli of smoothness can be used for characterization of weighted Besov spaces as well.

7. Second scale of weighted Besov spaces on $[-1,1]$. We introduce a second scale of weighted Besov spaces by using again as in $\S 6$ the kernels $\Phi_{j}$, defined by (4.1) with $\widehat{a}$ satisfying (4.2)-(4.3). 
Definition 7.1. Let $s \in \mathbb{R}$ and $0<p, q \leq \infty$. Then the weighted Besov space $\mathcal{B}_{p}^{s q}:=\mathcal{B}_{p}^{s q}(w)$ is defined as the set of all $f \in \mathcal{D}^{\prime}$ such that

$$
\|f\|_{\mathcal{B}_{p}^{s q}}:=\left(\sum_{j=0}^{\infty}\left[2^{s j}\left\|\mathcal{W}\left(2^{j} ; \cdot\right)^{-s} \Phi_{j} * f(\cdot)\right\|_{p}\right]^{q}\right)^{1 / q}<\infty,
$$

where the $l_{q}$-norm is replaced by the sup-norm if $q=\infty$.

As for the other weighted Besov and Triebel-Lizorkin spaces considered here the above definition is independent of the choice of $\widehat{a}$ satisfying (4.2)-(4.3). Also, the Besov space $\mathcal{B}_{p}^{s q}(w)$ is a quasi-Banach space which is continuously embedded in $\mathcal{D}^{\prime}$.

The main advantages of the spaces $\mathcal{B}_{p}^{s q}$ over $B_{p}^{s q}$ are that, first, they embed "correctly" with respect to the smoothness index $s$, and secondly, the right smoothness spaces in nonlinear $n$-term weighted approximation from needles are defined in terms of spaces $\mathcal{B}_{p}^{s q}$ (see $\S 8$ below).

Proposition 7.2. Let $0<p \leq p_{1}<\infty, 0<q \leq q_{1} \leq \infty$, and $-\infty<$ $s_{1} \leq s<\infty$. Then we have the continuous embedding

$$
\mathcal{B}_{p}^{s q} \subset \mathcal{B}_{p_{1}}^{s_{1} q_{1}} \quad \text { if } s-1 / p=s_{1}-1 / p_{1} .
$$

This embedding result follows readily by applying inequality (2.11).

We now define the sequence space $\mathbf{b}_{p}^{s q}(w)$ companion to $\mathcal{B}_{p}^{s q}(w)$.

Definition 7.3. Let $s \in \mathbb{R}$ and $0<p, q \leq \infty$. Then $\mathbf{b}_{p}^{s q}:=\mathbf{b}_{p}^{s q}(w)$ is defined to be the space of all complex-valued sequences $h:=\left\{h_{\xi}\right\}_{\xi \in \mathcal{X}}$ such that

$$
\|h\|_{\mathbf{b}_{p}^{s q}}:=\left(\sum_{j=0}^{\infty}\left[\sum_{\xi \in \mathcal{X}_{j}}\left(\mu\left(I_{\xi}\right)^{-s+1 / p-1 / 2}\left|h_{\xi}\right|\right)^{p}\right]^{q / p}\right)^{1 / q}<\infty
$$

with the usual modification for $p=\infty$ or $q=\infty$.

For the characterization of weighted Besov spaces $\mathcal{B}_{p}^{s q}$, we again employ the operators $S_{\varphi}$ and $T_{\psi}$ from (4.7). An argument similar to the proof of Lemma 4.4 shows that $T_{\psi}$ is well defined on $\mathbf{b}_{p}^{s q}$ (see also Lemma 6.3).

Theorem 7.4. Let $s \in \mathbb{R}$ and $0<p, q \leq \infty$. The operators $S_{\varphi}: \mathcal{B}_{p}^{s q} \rightarrow \mathbf{b}_{p}^{s q}$ and $T_{\psi}: \mathbf{b}_{p}^{s q} \rightarrow \mathcal{B}_{p}^{s q}$ are bounded and $T_{\psi} \circ S_{\varphi}=\operatorname{Id}$ on $\mathcal{B}_{p}^{s q}$. Consequently, for $f \in \mathcal{D}^{\prime}$ we have $f \in \mathcal{B}_{p}^{s q}$ if and only if $\left\{\left\langle f, \varphi_{\xi}\right\rangle\right\}_{\xi \in \mathcal{X}} \in \mathbf{b}_{p}^{s q}$. Moreover,

$$
\|f\|_{\mathcal{B}_{p}^{s q}} \sim\left\|\left\{\left\langle f, \varphi_{\xi}\right\rangle\right\}\right\|_{\mathbf{b}_{p}^{s q}} \sim\left(\sum_{j=0}^{\infty}\left[\sum_{\xi \in \mathcal{X}_{j}} \mu\left(I_{\xi}\right)^{-s p}\left\|\left\langle f, \varphi_{\xi}\right\rangle \psi_{\xi}\right\|_{p}^{p}\right]^{q / p}\right)^{1 / q} .
$$

In addition, the definition of $\mathcal{B}_{p}^{s q}$ is independent of the selection of $\widehat{a}$ satisfying (4.2)-(4.3).

The following additional lemma is needed for the proof of Theorem 7.4. 
Lemma 7.5. For every $P \in \Pi_{2^{j}}, j \geq 0$, and $0<p \leq \infty$,

$$
\left(\sum_{\xi \in \mathcal{X}_{j}} \mathcal{W}\left(2^{j} ; \xi\right)^{-s p} \sup _{x \in I_{\xi}}|P(x)|^{p} \mu\left(I_{\xi}\right)\right)^{1 / p} \leq c\left\|\mathcal{W}\left(2^{j} ; \cdot\right) P(\cdot)\right\|_{p} .
$$

The proof of this lemma is similar to the proof of Lemma 6.5, where one uses Lemma 5.4 in place of Lemma 4.10. We skip it.

For the proof of Theorem 7.4, one proceeds as in the proof of Theorem 6.4, using Lemma 7.5 instead of Lemma 6.5. The proof will be omitted.

\section{Application of weighted Besov spaces to nonlinear approx-} imation. We consider here nonlinear $n$-term approximation for a needlet system $\left\{\psi_{\eta}\right\}_{\eta \in \mathcal{X}}$ with $\varphi_{\eta}=\psi_{\eta}$, defined as in (3.5)-(3.8) with $\widehat{b}=\widehat{a}, \widehat{a} \geq 0$. Then $\widehat{a}$ satisfies

$$
\widehat{a}^{2}(t)+\widehat{a}^{2}(2 t)=1, \quad t \in[1 / 2,1] .
$$

Hence $\left\{\psi_{\eta}\right\}$ are real-valued.

Denote by $\Sigma_{n}$ the nonlinear set consisting of all functions $g$ of the form

$$
g=\sum_{\xi \in \Lambda} a_{\xi} \psi_{\xi}
$$

where $\Lambda \subset \mathcal{X}, \# \Lambda \leq n$, and $\Lambda$ is allowed to vary with $g$. Let $\sigma_{n}(f)_{p}$ denote the error of best $L^{p}(w)$-approximation to $f \in L^{p}(w)$ from $\Sigma_{n}$ :

$$
\sigma_{n}(f)_{p}:=\inf _{g \in \Sigma_{n}}\|f-g\|_{p} .
$$

The approximation will take place in $L^{p}(w), 0<p<\infty$. Assume in the following that $0<p<\infty, s>0$, and $1 / \tau:=s+1 / p$. We write briefly $\mathcal{B}_{\tau}^{s}:=\mathcal{B}_{\tau}^{s \tau}$.

By Theorem 7.4 and (3.12) it follows that

$$
\|f\|_{\mathcal{B}_{\tau}^{s}} \approx\left(\sum_{\xi \in \mathcal{X}}\left\|\left\langle f, \psi_{\xi}\right\rangle \psi_{\xi}\right\|_{p}^{\tau}\right)^{1 / \tau} .
$$

The embedding of $\mathcal{B}_{\tau}^{s}$ into $L^{p}(w)$ plays an important role here.

Proposition 8.1. If $f \in \mathcal{B}_{\tau}^{s}$, then $f$ can be identified as a function $f \in L^{p}(w)$ and

$$
\|f\|_{p} \leq\left\|\sum_{\xi \in \mathcal{X}}\left|\left\langle f, \psi_{\xi}\right\rangle \psi_{\xi}(\cdot)\right|\right\|_{p} \leq c\|f\|_{\mathcal{B}_{\tau}^{s}} .
$$

We now state our main result in this section.

TheOREM 8.2 (Jackson estimate). If $f \in \mathcal{B}_{\tau}^{s}$, then

$$
\sigma_{n}(f)_{p} \leq c n^{-s}\|f\|_{\mathcal{B}_{\tau}^{s}},
$$

where $c$ depends only on $s, p$, and the parameters of the needlet system. 
The proofs of this theorem and of Proposition 8.1 can be carried out exactly as the proofs of the respective Jackson estimate and embedding result in $[9,11]$ and will be omitted.

It is an open problem to prove the Bernstein estimate companion to $(8.3)$ :

$$
\|g\|_{\mathcal{B}_{\tau}^{s}} \leq c n^{s}\|g\|_{p} \quad \text { for } g \in \Sigma_{n}, 1<p<\infty .
$$

This would enable one to characterize the rates (approximation spaces) of nonlinear $n$-term approximation in $L^{p}(w)(1<p<\infty)$ from needlet systems.

\section{Proofs}

Proof of Proposition 2.2. We need the following integral representation of $L_{n}(x, y)$ from [13] (see $\left.(2.15)\right)$ :

$$
L_{n}(x, y)=c_{\alpha, \beta} \int_{0}^{\pi} \int_{0}^{1} L_{n}^{\alpha, \beta}(t(x, y, r, \psi)) d m_{\alpha, \beta}(r, \psi),
$$

where $L_{n}^{\alpha, \beta}(t)$ is defined by $(2.1)$,

$t(x, y, r, \psi):=\frac{1}{2}(1+x)(1+y)+\frac{1}{2}(1-x)(1-y) r^{2}+r \sqrt{1-x^{2}} \sqrt{1-y^{2}} \cos \psi-1$, the integral is against

$$
d m_{\alpha, \beta}(r, \psi):=\left(1-r^{2}\right)^{\alpha-\beta-1} r^{2 \beta+1}(\sin \psi)^{2 \beta} d r d \psi,
$$

and the constant $c_{\alpha, \beta}$ is determined from

$$
c_{\alpha, \beta} \int_{0}^{\pi} \int_{0}^{1} 1 d m_{\alpha, \beta}(r, \psi)=1 .
$$

For any $u \in[-1,1]$ we will denote by $\theta_{u}$ the only angle in $[0, \pi]$ such that $u=\cos \theta_{u}$.

We will need the following lemma contained in the proof of Theorem 2.4 in $[13]$.

Lemma 9.1. Let $\alpha, \beta>-1 / 2$ and $k \geq 2 \alpha+2 \beta+3$. Then there is a constant $c_{k}>0$ depending only on $k, \alpha$, and $\beta$ such that for $x, y \in[-1,1]$,

$$
\begin{aligned}
& \int_{0}^{\pi} \int_{0}^{1} \frac{n^{2 \alpha+1} d m_{\alpha, \beta}(r, \psi)}{(1+n \sqrt{1-t(x, y, r, \psi)})^{k}} \\
& \quad \leq c_{k} \frac{1}{\sqrt{\mathcal{W}_{\alpha, \beta}(n ; x)} \sqrt{\mathcal{W}_{\alpha, \beta}(n ; y)}\left(1+n\left|\theta_{x}-\theta_{y}\right|\right)^{\sigma}}
\end{aligned}
$$

where $\sigma=k-2 \alpha-2 \beta-3$. 
Identity (9.1) yields

$$
\begin{aligned}
& \left|L_{n}(x, y)-L_{n}(\xi, y)\right| \\
& \quad \leq c \int_{0}^{\pi}\left|L_{n}^{\alpha, \beta}(t(x, y, r, \psi))-L_{n}^{\alpha, \beta}(t(\xi, y, r, \psi))\right| d m_{\alpha, \beta}(r, \psi) \\
& \quad \leq c \int_{0}^{\pi} \int_{0}^{1}\left\|\partial L_{n}^{\alpha, \beta}(\cdot)\right\|_{L^{\infty}\left(I_{r, \psi}\right)}|t(x, y, r, \psi)-t(\xi, y, r, \psi)| d m_{\alpha, \beta}(r, \psi),
\end{aligned}
$$

where $\partial f=f^{\prime}$ and $I_{r, \psi}$ is the interval with end points $t(x, y, r, \psi)$ and $t(\xi, y, r, \psi)$.

From estimate (2.16) in [13] and Markov's inequality, for any $k$ there exists a constant $c_{k}>0$ such that

$$
\begin{aligned}
& \left\|\partial L_{n}^{\alpha, \beta}(\cdot)\right\|_{L^{\infty}\left(I_{r, \psi}\right)} \leq c_{k} \max _{u \in I_{r, \psi}} \frac{n^{2 \alpha+4}}{(1+n \sqrt{1-u})^{k}} \\
& \leq c_{k} n^{2 \alpha+4}\left[(1+n \sqrt{1-t(x, y, r, \psi)})^{-k}+(1+n \sqrt{1-t(\xi, y, r, \psi)})^{-k}\right] .
\end{aligned}
$$

For the rest of the proof we assume that $k>0$ is sufficiently large.

From the definition of $t(x, y, r, \psi)$ one easily obtains

which implies

$$
\begin{aligned}
1-t(x, y, r, \psi)= & 2 \sin ^{2} \frac{\theta_{x}-\theta_{y}}{2}+2 \sin ^{2} \frac{\theta_{x}}{2} \sin ^{2} \frac{\theta_{y}}{2}\left(1-r^{2}\right) \\
& +\sin \theta_{x} \sin \theta_{y}(1-r \cos \psi),
\end{aligned}
$$

$$
\begin{aligned}
& t(x, y, r, \psi)-t(\xi, y, r, \psi)=\cos \left(\theta_{\xi}-\theta_{y}\right)-\cos \left(\theta_{x}-\theta_{y}\right) \\
& \quad+\left(\cos \theta_{\xi}-\cos \theta_{x}\right) \sin ^{2} \frac{\theta_{y}}{2}\left(1-r^{2}\right)+\left(\sin \theta_{\xi}-\sin \theta_{x}\right) \sin \theta_{y}(1-r \cos \psi) .
\end{aligned}
$$

It is readily seen that

$$
\begin{aligned}
\left|\cos \left(\theta_{\xi}-\theta_{y}\right)-\cos \left(\theta_{x}-\theta_{y}\right)\right| & =2\left|\sin \frac{\theta_{x}+\theta_{\xi}-2 \theta_{y}}{2} \sin \frac{\theta_{\xi}-\theta_{x}}{2}\right| \\
& \leq\left|\theta_{\xi}-\theta_{x}\right|\left(\left|\theta_{z}-\theta_{y}\right|+c n^{-1}\right),
\end{aligned}
$$

where we used the inequalities $\left|\theta_{z}-\theta_{\xi}\right| \leq c n^{-1}$ and $\left|\theta_{x}-\theta_{\xi}\right| \leq c n^{-1}$. Therefore,

$$
\begin{aligned}
& |t(x, y, r, \psi)-t(\xi, y, r, \psi)| \\
\leq & \left|\theta_{\xi}-\theta_{x}\right|\left[\left(\left|\theta_{z}-\theta_{y}\right|+c n^{-1}\right)+\sin ^{2} \frac{\theta_{y}}{2}\left(1-r^{2}\right)+\sin \theta_{y}(1-r \cos \psi)\right] .
\end{aligned}
$$

We use this and (9.3) in (9.2) to obtain

$$
\left|L_{n}(x, y)-L_{n}(\xi, y)\right| \leq c\left|\theta_{\xi}-\theta_{x}\right|\left(A_{1}+B_{1}+A_{2}+B_{2}+A_{3}+B_{3}\right),
$$

where $A_{j}$ and $B_{j}$ are integrals of the same type with $A_{j}$ involving $t(x, y, r, \psi)$ and $B_{j}$ involving $t(\xi, y, r, \psi)$; the indices $j=1,2,3$ correspond to the three terms on the right-hand side of (9.4). We will estimate them separately. 
CASE 1. We first estimate the integral

$$
A_{1}:=n^{2 \alpha+4} \int_{0}^{\pi} \int_{0}^{1} \frac{\left|\theta_{z}-\theta_{y}\right|+c n^{-1}}{(1+n \sqrt{1-t(x, y, r, \psi)})^{k}} d m_{\alpha, \beta}(r, \psi)
$$

as well as the integral $B_{1}$, which is the same as $A_{1}$ but with $t(\xi, y, r, \psi)$ in place of $t(x, y, r, \psi)$.

Using the estimate in Lemma 9.1 and the fact that $\left|\theta_{z}-\theta_{y}\right| \sim\left|\theta_{x}-\theta_{y}\right|$ $+c n^{-1}$, we have

$$
\begin{aligned}
A_{1} & \leq c \frac{n^{3}\left(\left|\theta_{z}-\theta_{y}\right|+c n^{-1}\right)}{\sqrt{\mathcal{W}(n ; x)} \sqrt{\mathcal{W}(n ; y)}\left(1+n\left|\theta_{x}-\theta_{y}\right|\right)^{\sigma}} \\
& \leq c \frac{n^{2}}{\sqrt{\mathcal{W}(n ; x)} \sqrt{\mathcal{W}(n ; y)}\left(1+n\left|\theta_{z}-\theta_{y}\right|\right)^{\sigma-1}} .
\end{aligned}
$$

On account of (2.22) this gives the desired estimate.

The integral $B_{1}$ is estimated similarly with the same bound.

CASE 2. We now estimate the integrals

$$
A_{2}:=n^{2 \alpha+4} \int_{0}^{\pi} \int_{0}^{1} \frac{\sin ^{2} \frac{\theta_{y}}{2}\left(1-r^{2}\right)}{(1+n \sqrt{1-t(x, y, r, \psi)})^{k}} d m_{\alpha, \beta}(r, \psi)
$$

and $B_{2}$ which is the same but has $t(\xi, y, r, \psi)$ in place of $t(x, y, r, \psi)$.

By the definition of $d m_{\alpha, \beta}(r, \psi),\left(1-r^{2}\right) d m_{\alpha, \beta}(r, \psi)=d m_{\alpha+1, \beta}(r, \psi)$. Then using the estimate from Lemma 9.1 with $\alpha$ replaced by $\alpha+1$, we get

$$
\begin{aligned}
A_{2} & \leq c \frac{n \sin ^{2} \frac{y}{2}}{\sqrt{\mathcal{W}_{\alpha+1, \beta}(n ; x)} \sqrt{\mathcal{W}_{\alpha+1, \beta}(n ; y)}\left(1+n\left|\theta_{x}-\theta_{y}\right|\right)^{\sigma}} \\
& \leq c \frac{n^{2}}{\sqrt{\mathcal{W}_{\alpha, \beta}(n ; x)} \sqrt{\mathcal{W}_{\alpha, \beta}(n ; y)}\left(1+n\left|\theta_{x}-\theta_{y}\right|\right)^{\sigma}},
\end{aligned}
$$

where we used the fact that $\mathcal{W}_{\alpha+1, \beta}(n ; y)=\mathcal{W}_{\alpha, \beta}(n ; y)\left(\sin ^{2}\left(\theta_{y} / 2\right)+n^{-2}\right)$ and hence $\mathcal{W}_{\alpha+1, \beta}(n ; x) \geq \mathcal{W}_{\alpha, \beta}(n ; x) n^{-2}$. The equivalence $\left|\theta_{z}-\theta_{y}\right| \sim\left|\theta_{x}-\theta_{y}\right|$ $+c n^{-1}$ and (2.22) then give the desired estimate. The integral $B_{2}$ is estimated similarly.

CASE 3. We finally estimate the integrals

$$
A_{3}:=n^{2 \alpha+4} \int_{0}^{\pi} \int_{0}^{1} \frac{\sin \theta_{y}(1-r \cos \psi)}{(1+n \sqrt{1-t(x, y, r, \psi)})^{k}} d m_{\alpha, \beta}(r, \psi)
$$

and $B_{3}$ which has $t(\xi, y, r, \psi)$ in place of $t(x, y, r, \psi)$.

Assume first that $\left|\sin \theta_{x}\right| \geq n^{-1}$. Using the fact that

$$
1-t(x, y, r, \psi) \geq \sin \theta_{x} \sin \theta_{y}(1-r \cos \psi),
$$


we conclude that

$$
\begin{aligned}
A_{3} & \leq \frac{n^{2 \alpha+2}}{\sin \theta_{x}} \int_{0}^{\pi} \int_{0}^{1} \frac{1}{(1+n \sqrt{1-t(x, y, r, \psi)})^{k-2}} d m_{\alpha, \beta}(r, \psi) \\
& \leq c n^{2 \alpha+3} \int_{0}^{\pi} \int_{0}^{1} \frac{1}{(1+n \sqrt{1-t(x, y, r, \psi)})^{k-2}} d m_{\alpha, \beta}(r, \psi) .
\end{aligned}
$$

Now the estimate from Lemma 9.1 can be applied to get the desired estimate.

Let $\left|\sin \theta_{x}\right| \leq n^{-1}$. We have

$$
\left|\sin \theta_{y}\right| \leq\left|\sin \theta_{y}-\sin \theta_{x}\right|+\left|\sin \theta_{x}\right| \leq\left|\theta_{y}-\theta_{x}\right|+n^{-1}
$$

and use the fact that

$$
1-t(x, y, r, \psi) \geq 2 \sin ^{2} \frac{\theta_{x}-\theta_{y}}{2} \geq c\left(\theta_{x}-\theta_{y}\right)^{2}
$$

to conclude that

$$
A_{3} \leq c n^{2 \alpha+3} \int_{0}^{\pi} \int_{0}^{1} \frac{1}{(1+n \sqrt{1-t(x, y, r, \psi}))^{k-2}} d m_{\alpha, \beta}(r, \psi) .
$$

Applying the estimate from Lemma 9.1 we obtain the desired result. $B_{3}$ is estimated in the same way.

Putting the above estimates together completes the proof of Theorem 2.2 .

Proof of Proposition 2.4. Note first that it suffices to prove (2.6) only for $n \geq n_{0}$, where $n_{0}$ is sufficiently large. This follows from the fact that $P_{n}^{(\alpha, \beta)}$ and $P_{n+1}^{(\alpha, \beta)}$ do not have common zeros and $\mathcal{W}(n ; x) \sim 1$ if $n \leq$ const. Furthermore, since $P_{k}^{(\alpha, \beta)}(-x)=(-1)^{k} P_{k}^{(\beta, \alpha)}(x)$, it is sufficient to consider only the case $x \in[0,1]$.

Note that the Jacobi polynomials are normalized by $P_{k}^{(\alpha, \beta)}(1)=\left(\begin{array}{c}k+\alpha \\ k\end{array}\right)$ $\sim k^{\alpha}$ and using Markov's inequality it follows that $P_{k}^{(\alpha, \beta)}(x) \geq c k^{\alpha}$ for $1-\delta k^{-2} \leq x \leq 1$, where $\delta>0$ is a sufficiently small constant. From this one readily infers that $(2.6)$ holds for $1-\delta_{1} n^{-2} \leq x \leq 1, \delta_{1}>0$. Define $\theta \in[0, \pi]$ from $x=\cos \theta$. Then the latter condition on $x$ is apparently equivalent to $0 \leq \theta \leq \delta_{2} n^{-1}$ with $\delta_{2}$ being a positive constant.

To estimate $\Lambda_{n}(\cos \theta)$ for $c^{*} n^{-1} \leq \theta \leq \pi / 2$ with $c^{*}>0$ sufficiently large, we need the following asymptotic formula of the Jacobi polynomials: For $\alpha, \beta>-1$,

$$
\begin{aligned}
\left(\sin \frac{\theta}{2}\right)^{\alpha}\left(\cos \frac{\theta}{2}\right)^{\beta} P_{n}^{(\alpha, \beta)}(\cos \theta)= & N^{-\alpha} \frac{\Gamma(n+\alpha+1)}{n !}\left(\frac{\theta}{\sin \theta}\right)^{1 / 2} J_{\alpha}(N \theta) \\
& +\theta^{1 / 2} \mathcal{O}\left(n^{-3 / 2}\right)
\end{aligned}
$$


if $c_{0} n^{-1} \leq \theta \leq \pi / 2$, where $N=n+\eta$ with $\eta:=(\alpha+\beta+1) / 2, J_{\alpha}$ is the Bessel function, and $c_{0}>0$ is an arbitrary but fixed constant (see [18, Theorem 8.21.12, p. 195]).

Since $2 / \pi \leq(\sin \theta) / \theta \leq 1$ and $(\cos \theta) / 2 \sim 1$ on $[0, \pi / 2]$, and also $\Gamma(n+\alpha+1) / n ! \sim n^{\alpha}$, we infer from the above that

$$
\left(\sin \frac{\theta}{2}\right)^{2 \alpha}\left[P_{k}^{(\alpha, \beta)}(\cos \theta)\right]^{2} \geq c_{1}\left[J_{\alpha}((k+\eta) \theta)\right]^{2}-c_{2} k^{-3 / 2} \theta^{1 / 2}\left|J_{\alpha}((k+\eta) \theta)\right| .
$$

Recall the well known asymptotic formula

$$
J_{\alpha}(z)=\left(\frac{2}{\pi z}\right)^{1 / 2}\left[\cos (z+\gamma)+O\left(z^{-1}\right)\right], \quad z \rightarrow \infty,
$$

where $\gamma=-\alpha \pi / 2-\pi / 4$.

All of the above leads to

$$
\begin{aligned}
\left(\sin \frac{\theta}{2}\right)^{2 \alpha} & \Lambda_{n}(\cos \theta) \\
\geq & \sum_{k=n}^{n+[\varepsilon n]}\left(c_{1}\left[J_{\alpha}((k+\eta) \theta)\right]^{2}-c_{2} k^{-3 / 2} \theta^{1 / 2}\left|J_{\alpha}((k+\eta) \theta)\right|\right) \\
\geq & \frac{c}{n \theta} \sum_{k=n}^{n+[\varepsilon n]}\left[\cos ^{2}(k \theta+b(\theta))-c^{\prime}(n \theta)^{-1}\right]-c^{\prime \prime} \varepsilon n^{-1},
\end{aligned}
$$

for $c_{0} n^{-1} \leq \theta \leq \pi / 2$, where $b(\theta)=((\alpha+\beta+1) / 2) \theta+\gamma$. We now use the well known identities for the Dirichlet kernel and its conjugate to obtain, for $m>n$,

$$
\sum_{k=n}^{m} \cos ^{2}(k \theta+b)=\frac{1}{2}(m-n+1)+(\cos 2 b+\sin 2 b) \frac{\sin (m-n+1) \theta \cos (n+m) \theta}{2 \sin \theta} .
$$

Therefore,

$$
\begin{aligned}
\sum_{k=n}^{n+[\varepsilon n]} \cos ^{2}(k \theta+b(\theta)) & \geq \frac{1}{2}([\varepsilon n]+1)\left(1-\frac{2}{([\varepsilon n]+1) \sin \theta}\right) \\
& \geq \frac{1}{2} \varepsilon n\left(1-\frac{\pi}{\varepsilon n \theta}\right) \geq \frac{\varepsilon n}{4},
\end{aligned}
$$

whenever $(2 \pi / \varepsilon) n^{-1} \leq \theta \leq \pi / 2$. Substituting this in (9.5) we obtain

$$
\begin{aligned}
\left(\sin \frac{\theta}{2}\right)^{2 \alpha} \Lambda_{n}(\cos \theta) & \geq \frac{c}{n \theta}\left(\frac{\varepsilon n}{4}-\frac{c^{\prime} \varepsilon n}{n \theta}\right)-\frac{c^{\prime \prime} \varepsilon}{n} \\
& \geq \frac{c \varepsilon}{\theta}\left(\frac{1}{4}-\frac{c^{\prime}}{c^{*}}\right)-\frac{c^{\prime \prime} \varepsilon}{n} \geq \frac{c^{\diamond}}{\theta}, \quad c^{\diamond}>0,
\end{aligned}
$$


if $c^{*} n^{-1} \leq \theta \leq \pi / 2$ with $c^{*}:=\max \left\{c_{0}, 8 c^{\prime}, 2 \pi / \varepsilon\right\}$ and $n$ is sufficiently large. Hence,

$$
\Lambda_{n}(\cos \theta) \geq \frac{c^{\diamond}}{\theta}\left(\sin \frac{\theta}{2}\right)^{-2 \alpha} \geq c \theta^{-(2 \alpha+1)}, \quad c^{*} n^{-1} \leq \theta \leq \pi / 2,
$$

for sufficiently large $n$, which yields (2.6) in this case.

For the remaining case $\delta_{2} n^{-1} \leq \theta \leq c^{*} n^{-1}$, we need further properties of Jacobi polynomials. Let $x_{\nu, n}=\cos \theta_{\nu, n}$ denote the zeros of the Jacobi polynomial $P_{n}^{(\alpha, \beta)}$, where

$$
0<\theta_{1, n}<\theta_{2, n}<\cdots<\theta_{n, n}<\pi .
$$

It is well known that $\theta_{\nu, n} \sim \nu / n$, but we will need a much more precise asymptotic representation for $\theta_{\nu, n}$ (see below). The Jacobi polynomials satisfy the following relation (see e.g. [12, Theorem 3.3, p. 171]):

$$
P_{n}^{(\alpha, \beta)}(\cos \theta) \sim n^{1 / 2}\left|\theta-\theta_{\nu_{\theta}, n}\right| \frac{n^{\alpha+1 / 2}}{\nu_{\theta}^{\alpha+1 / 2}}, \quad \theta \in[0, \pi],
$$

where $\nu_{\theta}$ denotes the index of the zero $x_{\nu, n}, 1 \leq \nu \leq n$, which is (one of) the closest to $x(x=\cos \theta)$.

We will need the asymptotics of the zeros of the Jacobi polynomials from [5]:

$$
\theta_{\nu, n}=\frac{j_{\alpha, \nu}}{N}+\frac{1}{4 N^{2}}\left[\left(\alpha^{2}-\frac{1}{4}\right) \frac{1-t \cot t}{2 t}-\frac{\alpha^{2}-\beta^{2}}{4} \tan \frac{t}{2}\right]+t^{2} \mathcal{O}\left(n^{-3}\right),
$$

where $N=n+\eta$ as before, $j_{\alpha, \nu}$ is the $\nu$ th positive zero of the Bessel function $J_{\alpha}(x)$ and $t=j_{\alpha, \nu} / N$. Here the $\mathcal{O}$-term is uniformly bounded for $\nu=1, \ldots,[\gamma n]$, where $\gamma \in(0,1)$. It is easy to verify that $(1-t \cot t) / t=\mathcal{O}(t)$ as $t \rightarrow 0$ and obviously $1 /(n+\eta)-1 / n=\mathcal{O}\left(n^{-2}\right)$. Hence

$$
\theta_{\nu, n}=\frac{j_{\alpha, \nu}}{n}+\mathcal{O}\left(n^{-2}\right), \quad \nu=1, \ldots,[\gamma n] .
$$

We will also use the fact that

$$
0<j_{\alpha, 1}<j_{\alpha, 2}<\cdots \quad \text { and } j_{\alpha, \nu} \rightarrow \infty .
$$

Let $j_{\alpha, \nu_{\max }}:=\max \left\{j_{\alpha, \nu}: j_{\alpha, \nu} \leq(1+\varepsilon) c^{*}\right\}$ and $\mathcal{J}:=\left\{j_{\alpha, 1}, j_{\alpha, 2}, \ldots, j_{\alpha, \nu_{\max }}\right\}$. Notice that $\nu_{\max }$ is a constant independent of $n$. Suppose that $\mathcal{J} \neq \emptyset$ (the case $\mathcal{J}=\emptyset$ is easier).

Fix $\delta_{2} n^{-1} \leq \theta \leq c^{*} n^{-1}$. Then by (9.8) it follows that

$$
P_{k}^{(\alpha, \beta)}(\cos \theta) \sim k^{\alpha+1}\left|\theta-\theta_{\nu_{\theta}, k}\right|,
$$

where the $\nu_{\theta}$ 's involved are bounded by a constant independent of $n$. Hence, (9.10) can be used to represent $\theta_{\nu_{\theta}, k}$ for $n \leq k \leq n+[\varepsilon n]$ if $n$ is sufficiently 
large. Using the above we get

$$
\begin{aligned}
\Lambda_{n}(\cos \theta) & \geq c n^{2 \alpha+2} \sum_{k=n}^{n+[\varepsilon n]}\left|\theta-\theta_{\nu_{\theta}, k}\right|^{2} \geq c n^{2 \alpha} \sum_{k=n}^{n+[\varepsilon n]}\left|k \theta-k \theta_{\nu_{\theta}, k}\right|^{2} \\
& \geq c n^{2 \alpha} \sum_{k=n}^{n+[\varepsilon n]}\left(\left|k \theta-j_{\alpha, \nu_{\theta}}\right|^{2}-c^{\prime} k^{-1}\left|k \theta-j_{\alpha, \nu_{\theta}}\right|\right) \\
& \geq c n^{2 \alpha}\left(\sum_{k=n}^{n+[\varepsilon n]}\left|k \theta-j_{\alpha, \nu_{\theta}}\right|^{2}-c^{\prime} c^{*} \varepsilon\right),
\end{aligned}
$$

where we used (9.10). Therefore,

$$
\Lambda_{n}(\cos \theta) \geq c n^{2 \alpha}\left(\sum_{k=n}^{n+[\varepsilon n]} \operatorname{dist}(k \theta, \mathcal{J})^{2}-c^{\diamond}\right), \quad c, c^{\diamond}>0,
$$

where $\operatorname{dist}(k \theta, \mathcal{J})$ denotes the distance of $k \theta$ from the set $\mathcal{J}$, that is, the distance of $k \theta$ from the nearest zero of the Bessel function $J_{\alpha}(x)$.

It remains to estimate the sum in (9.11). Define $\mathcal{K}:=\{n, n+1, \ldots$, $n+[\varepsilon n]\}$ and let $\mathcal{K}_{0}$ be the set of all indices $k \in \mathcal{K}$ such that $\operatorname{dist}(k \theta, \mathcal{J})<m \theta$, where $m:=\left[\varepsilon n /\left(6 \nu_{\max }\right)\right]$. Evidently

$\# \mathcal{K}_{0} \leq(2 m+1) \nu_{\max } \leq\left(2\left[\varepsilon n /\left(6 \nu_{\max }\right)\right]+1\right) \nu_{\max } \leq \varepsilon n / 2 \quad$ if $n \geq 6 \nu_{\max } \varepsilon^{-1}$.

Then $\# \mathcal{K} \backslash \mathcal{K}_{0} \geq[\varepsilon n]+1-\varepsilon n / 2 \geq \varepsilon n / 2$ and hence

$$
\sum_{k=n}^{n+[\varepsilon n]} \operatorname{dist}(k \theta, \mathcal{J})^{2} \geq \sum_{k \in \mathcal{K} \backslash \mathcal{K}_{0}}(m \theta)^{2} \geq c \sum_{k \in \mathcal{K} \backslash \mathcal{K}_{0}}(n \theta)^{2} \geq c \delta_{2}^{2} \varepsilon n \geq c_{*} n, \quad c_{*}>0 .
$$

Inserting this in (9.11) we obtain

$$
\Lambda_{n}(\cos \theta) \geq c n^{2 \alpha}\left(c_{*} n-c^{\diamond}\right) \geq \widetilde{c} n^{2 \alpha+1}
$$

for sufficiently large $n$. This implies the stated inequality (2.6) with $x=\cos \theta$ in the case $\delta_{2} n^{-1} \leq \theta \leq c^{*} n^{-1}$. The proof of Proposition 2.4 is complete.

Proof of Proposition 2.6. Suppose $\alpha \geq \beta$ and let $1<q<\infty$. By Lemma 2.5(i) we have $g=L_{n} * g$ and using Hölder's inequality, (2.3), and the fact that $\mathcal{W}(n ; x) \geq c n^{-2 \alpha-1}$ we obtain

$$
|g(x)| \leq c\|g\|_{q}\left(\frac{n}{\mathcal{W}(n ; x)}\right)^{1 / q} \leq c n^{(2 \alpha+2) / q}\|g\|_{q}, \quad x \in[-1,1],
$$

which leads to

$$
\|g\|_{\infty} \leq c n^{(2 \alpha+2) / q}\|g\|_{q}, \quad 1<q \leq \infty
$$


If $0<q \leq 1$, then the above inequality with $q=2$ gives

$$
\|g\|_{\infty}^{2} \leq c n^{2 \alpha+2} \int_{-1}^{1}|g(y)|^{2-q}|g(y)|^{q} w(y) d y \leq c n^{2 \alpha+2}\|g\|_{\infty}^{2-q}\|g\|_{q}^{q},
$$

which shows that (9.12) holds for $0<q \leq 1$ as well.

Let $0<q<p<\infty$ (the case $p=\infty$ is contained in (9.12)). Then using (9.12) we obtain

$$
\begin{aligned}
\|g\|_{p} & =\left(\int_{-1}^{1}|g(x)|^{p-q}|g(x)|^{q} w(x) d x\right)^{1 / p} \\
& \leq c n^{(2 \alpha+2)(1 / q-1 / p)}\|g\|_{q}^{(p-q) / p}\|g\|_{q}^{q / p}=c n^{(2 \alpha+2)(1 / q-1 / p)}\|g\|_{q} .
\end{aligned}
$$

On the other hand, by [12, p. 114],

$$
\|g\|_{p} \leq c n^{2(1 / q-1 / p)}\|g\|_{q} .
$$

Putting the above two estimates together gives (2.10).

To prove (2.11) we will need the following inequality:

$$
\begin{aligned}
& \int_{-1}^{1} \frac{w(y)}{\mathcal{W}(n ; y)^{p / 2+\gamma}(1+n d(x, y))^{\sigma}} d y \\
& \quad \leq c \frac{1}{n \mathcal{W}(n ; x)^{p / 2+\gamma-1}}, \quad x \in[-1,1],
\end{aligned}
$$

where $\gamma \in \mathbb{R}$ and $\sigma$ is sufficiently large. The proof of (9.13) is contained in the proof of Proposition 1 in [13]. Assume $1<q<\infty$. Then using Lemma 2.5(i), Hölder's inequality $\left(1 / q+1 / q^{\prime}=1\right)$, and (1.10) we have, for $x \in[-1,1]$,

$$
\begin{aligned}
|g(x)| \leq & \left\|\mathcal{W}(n ; \cdot)^{s+1 / p-1 / q} g(\cdot)\right\|_{q} \\
& \times\left(\int_{-1}^{1}\left|L_{n}(x, y) \mathcal{W}(n ; y)^{-s-1 / p+1 / q}\right|^{q^{\prime}} w(y) d y\right)^{1 / q^{\prime}} \\
\leq & c \frac{n}{\mathcal{W}(n ; x)^{1 / 2}}\left(\int_{-1}^{1} \frac{w(y) d y}{\mathcal{W}(n ; y)^{q^{\prime} / 2+\gamma}(1+n d(x, y))^{\sigma}}\right)^{1 / q^{\prime}} \\
& \times\left\|\mathcal{W}(n ; \cdot)^{s+1 / p-1 / q} g(\cdot)\right\|_{q}
\end{aligned}
$$

with $\gamma=q^{\prime}(s+1 / p-1 / q)$. Now, applying (9.13) we infer that

$$
|g(x)| \leq c \frac{n^{1 / q}}{\mathcal{W}(n ; x)^{s+1 / p}}\left\|\mathcal{W}(n ; \cdot)^{s+1 / p-1 / q} g(\cdot)\right\|_{q},
$$

which implies

$$
\left\|\mathcal{W}(n ; \cdot)^{s+1 / p} g(\cdot)\right\|_{\infty} \leq c n^{1 / q}\left\|\mathcal{W}(n ; \cdot)^{s+1 / p-1 / q} g(\cdot)\right\|_{q}, \quad 1<q \leq \infty .
$$


If $0<q \leq 1$, then by (9.14) with $q=2$,

$$
\begin{aligned}
\left\|\mathcal{W}(n ; \cdot)^{s+1 / p} g(\cdot)\right\|_{\infty} & \leq c n^{1 / 2}\left\|\mathcal{W}(n ; \cdot)^{s+1 / p-1 / 2} g(\cdot)\right\|_{2} \\
& \leq c n^{1 / 2}\left\|\mathcal{W}(n ; \cdot)^{s+1 / p} g(\cdot)\right\|_{\infty}^{1-q / 2}\left\|\mathcal{W}(n ; \cdot)^{s+1 / p-1 / q} g(\cdot)\right\|_{q}^{q / 2},
\end{aligned}
$$

which shows that (9.14) holds for $0<q \leq 1$ as well. Let $p<\infty$. Using (9.14), we get

$$
\begin{aligned}
& \left\|\mathcal{W}(n ; \cdot)^{s} g(\cdot)\right\|_{p}=\left(\int_{-1}^{1}\left|\mathcal{W}(n ; x)^{s} g(x)\right|^{p-q}\left|\mathcal{W}(n ; x)^{s} g(x)\right|^{q} w(x) d x\right)^{1 / p} \\
& \quad \leq c n^{1 / q-1 / p}\left\|\mathcal{W}(n ; \cdot)^{s+1 / p-1 / q} g(\cdot)\right\|_{q}^{1-q / p}\left(\int_{-1}^{1} \frac{\left|\mathcal{W}(n ; x)^{s} g(x)\right|^{q}}{\mathcal{W}(n ; x)^{(p-q) / p}} w(x) d x\right)^{1 / p} \\
& \quad=c n^{1 / q-1 / p}\left\|\mathcal{W}(n ; \cdot)^{s+1 / p-1 / q} g(\cdot)\right\|_{q} .
\end{aligned}
$$

Thus (2.11) is established if $p<\infty$. When $p=\infty$, (2.11) follows from (9.14).

Proof of estimate (2.22). We only consider the case when $-1 / 2 \leq x, y$ $\leq 1$ since the other cases are similar or simpler. Choose $\theta, \phi \in[0,2 \pi / 3]$ so that $x=\cos \theta, y=\cos \phi$. Then $d(x, y)=|\theta-\phi|$. We have

$$
\begin{aligned}
\sin \theta+n^{-1} & \leq|\sin \theta-\sin \phi|+\sin \phi+n^{-1} \leq|\theta-\phi|+\sin \phi+n^{-1} \\
& \leq(1+n|\theta-\phi|)\left(\sin \phi+n^{-1}\right) .
\end{aligned}
$$

Then (2.22) follows using (2.20).

Proof of Lemma 2.7. We first show that for $y \in[0,1]$ and $0<r \leq \pi$,

$$
\mu\left(B_{y}(r)\right):=\int_{B_{y}(r)} w(x) d x \sim r(d(y, 1)+r)^{2 \alpha+1} .
$$

Indeed, choose $0 \leq \zeta \leq \pi / 2$ so that $y=\cos \zeta$ and consider the case when $\zeta+r \leq 2 \pi / 3$ (the case $\zeta+r>2 \pi / 3$ is trivial; then $\mu\left(B_{y}(r)\right) \sim 1$ ). We have

$$
\begin{aligned}
\mu\left(B_{y}(r)\right) & \sim \int_{\max \{\zeta-r, 0\}}^{\zeta+r}(1-\cos u)^{\alpha} \sin u d u \sim \int_{\max \{\zeta-r, 0\}}^{\zeta+r} u^{2 \alpha+1} d u \\
& \sim(\zeta+r-\max \{\zeta-r, 0\})(\zeta+r)^{2 \alpha+1} \sim r(\zeta+r)^{2 \alpha+1},
\end{aligned}
$$

which yields (9.15).

We now proceed with the proof of $(2.25)$. Write $J_{\eta}:=B_{\eta}(\varepsilon)$, which is an interval. Equivalence (2.25) is trivial when $x \in J_{\eta}$.

Assume $x \in[-1,1] \backslash J_{\eta}$. Denote by $I_{x, \eta}$ the interval with end points $x$ and $\eta$. By the definition of the maximal operator in (2.23) it readily follows 
that

$$
\left(\frac{\mu\left(J_{\eta}\right)}{\mu\left(J_{\eta} \cup I_{x, \eta}\right)}\right)^{1 / t} \leq\left(\mathcal{M}_{t} \mathbb{1}_{J_{\eta}}\right)(x) \leq\left(\frac{\mu\left(J_{\eta}\right)}{\mu\left(I_{x, \eta}\right)}\right)^{1 / t},
$$

and since $\mu\left(I_{x, \eta}\right) \leq \mu\left(J_{\eta} \cup I_{x, \eta}\right) \leq \mu\left(J_{\eta}\right)+\mu\left(I_{x, \eta}\right) \leq c \mu\left(I_{x, \eta}\right)$, we have

$$
\left(\mathcal{M}_{t} \mathbb{1}_{J_{\eta}}\right)(x) \sim\left(\frac{\mu\left(J_{\eta}\right)}{\mu\left(I_{x, \eta}\right)}\right)^{1 / t} .
$$

We will only consider the case when $x \in[-1 / 2,1]$, since the case $x \in$ $[-1,-1 / 2]$ is simpler. Choose $\gamma \in[0, \pi / 2]$ and $\phi \in[0,2 \pi / 3]$ so that $\eta=\cos \gamma$ and $x=\cos \phi$. Then $d(\eta, x)=|\gamma-\phi|$. By (9.15) and (9.16) it follows that

$$
\begin{aligned}
{\left[\left(\mathcal{M}_{t} \mathbb{1}_{J_{\eta}}\right)(x)\right]^{t} } & \sim \frac{\varepsilon(d(\eta, 1)+\varepsilon)^{2 \alpha+1}}{\frac{|\gamma-\phi|}{2}\left(\frac{\gamma+\phi}{2}+\frac{|\gamma-\phi|}{2}\right)^{2 \alpha+1}} \\
& \sim \frac{\varepsilon}{d(\eta, x)}\left(\frac{d(\eta, 1)+\varepsilon}{d(\eta, x)+d(\eta, 1)}\right)^{2 \alpha+1},
\end{aligned}
$$

which implies (2.25). Estimates (2.26) are immediate from (2.25).

Proof of (3.12)-(3.13). The equivalence $\left\|\widetilde{\mathbb{1}}_{I_{\xi}}\right\|_{p} \sim\left(2^{-j} \mathcal{W}\left(2^{j} ; \xi\right)\right)^{1 / p-1 / 2}$ follows from (2.19).

From (2.3) and (2.15) it follows that, for $\xi \in \mathcal{X}_{j}$ and $0<p<\infty$,

$$
\left\|\varphi_{\xi}\right\|_{p},\left\|\psi_{\xi}\right\|_{p} \leq c c_{\xi}^{1 / 2}\left(\frac{2^{j}}{\mathcal{W}\left(2^{j} ; \xi\right)}\right)^{1-1 / p} \leq c\left(\frac{2^{j}}{\mathcal{W}\left(2^{j} ; \xi\right)}\right)^{1 / 2-1 / p} .
$$

When $p=\infty$, similar estimates follow from (3.11).

To estimate $\left\|\varphi_{\xi}\right\|_{p},\left\|\psi_{\xi}\right\|_{p}$ from below, we first note that from (2.5) and (9.17) it follows that $\left\|\varphi_{\xi}\right\|_{2} \sim\left\|\psi_{\xi}\right\|_{2} \sim 1$. Let $2<p<\infty$ and $1 / p+1 / p^{\prime}=1$. Using Hölder's inequality and (9.17) we obtain

$$
0<c \leq\left\|\varphi_{\xi}\right\|_{2}^{2} \leq\left\|\varphi_{\xi}\right\|_{p}\left\|\varphi_{\xi}\right\|_{p^{\prime}} \leq\left\|\varphi_{\xi}\right\|_{p}\left(\frac{2^{j}}{\mathcal{W}\left(2^{j} ; \xi\right)}\right)^{1 / 2-1 / p^{\prime}}
$$

and similarly for $\psi$. Hence

$$
\left\|\varphi_{\xi}\right\|_{p},\left\|\psi_{\xi}\right\|_{p} \geq c\left(\frac{2^{j}}{\mathcal{W}\left(2^{j} ; \xi\right)}\right)^{1 / 2-1 / p} .
$$

In the case $p=\infty$, we proceed similarly and obtain the same estimate.

If $0<p<2$, then

$$
0<c \leq\left\|\varphi_{\xi}\right\|_{2}^{2} \leq\left\|\varphi_{\xi}\right\|_{p}^{p}\left\|\varphi_{\xi}\right\|_{\infty}^{2-p} \leq c\left\|\varphi_{\xi}\right\|_{p}^{p}\left(\frac{2^{j}}{\mathcal{W}\left(2^{j} ; \xi\right)}\right)^{1-p / 2},
$$

which implies (9.18) and similarly for $\left\|\psi_{\xi}\right\|_{p}$.

Finally, (3.13) follows from the lower bound in (9.18) with $p=\infty$ and (3.10). 
Proof of Lemma 4.6. Estimate (4.13) is immediate from (3.11) and Lemma 2.7 (see (2.26) and (2.19)).

For the proof of (4.14) we first observe that by (3.13) there exists a point $\zeta \in B_{\xi}\left(c^{*} 2^{-j}\right)$ such that $\left|\varphi_{\xi}(\zeta)\right| \geq c^{\diamond}\left(2^{j} / \mathcal{W}\left(2^{j} ; \xi\right)\right)^{1 / 2}$. By (2.4) it follows that

$$
\left|\varphi_{\xi}(x)-\varphi_{\xi}(\zeta)\right| \leq c \frac{2^{3 j / 2} d(x, \zeta)}{\sqrt{\mathcal{W}\left(2^{j} ; \xi\right)}}, \quad x \in B_{\zeta}\left(c 2^{-j}\right),
$$

and hence for sufficiently small constant $c^{b}>0$ we have

$$
\left|\varphi_{\xi}(x)\right| \geq \frac{c^{\diamond}}{2}\left(2^{j} / \mathcal{W}\left(2^{j} ; \xi\right)\right)^{1 / 2} \quad \text { for } x \in B_{\zeta}\left(c^{\natural} 2^{-j}\right) \cap[-1,1] .
$$

Therefore, there exists an interval $J_{\eta}:=B_{\eta}\left(c 2^{-j}\right) \subset[-1,1]$ such that

$$
\left\|\varphi_{\xi}\right\|_{L^{\infty}\left(J_{\eta}\right)} \geq c\left(2^{j} / \mathcal{W}\left(2^{j} ; \xi\right)\right)^{1 / 2} \text { and } d(\eta, \xi) \leq c 2^{-j} .
$$

Hence,

$$
\left(\mathcal{M}_{t} \varphi_{\xi}\right)(x) \geq c\left(\mathcal{M}_{t} \widetilde{\mathbb{1}}_{J_{\eta}}\right)(x) \geq c \widetilde{\mathbb{1}}_{I_{\xi}}(x),
$$

where for the last estimate we used (2.25). Thus (4.14) is established for $\varphi_{\xi}$. The proof for $\psi_{\xi}$ is the same.

Proof of Lemma 4.7. From the orthogonality of Jacobi polynomials it follows that $\Phi_{j} * \psi_{\xi}(x)=0$ if $\xi \in \mathcal{X}_{\nu}$, where $\nu \geq j+2$ or $\nu \leq j-2$.

Assume that $\xi \in \mathcal{X}_{\nu}, j-1 \leq \nu \leq j+1$. From the localization of the kernels $\Phi_{j}, \Psi_{\nu}$ (see (3.9)) and the definition of $c_{\xi}$ (see (2.14)) we see that for any $\sigma>0$ there is a constant $c_{\sigma}>0$ such that

$$
\begin{aligned}
& \left|\Phi_{j} * \psi_{\xi}(x)\right|=\sqrt{c_{\xi}}\left|\int_{-1}^{1} \Phi_{j}(x, y) \Psi_{\nu}(y, \xi) w(y) d y\right| \\
& \leq c 2^{3 j / 2} \mathcal{W}\left(2^{j} ; x\right)^{-1 / 2} \int_{-1}^{1} \frac{w(y)}{\mathcal{W}\left(2^{j} ; y\right)\left(1+2^{j} d(x, y)\right)^{\sigma}\left(1+2^{j} d(y, \xi)\right)^{\sigma}} d y .
\end{aligned}
$$

Setting $\xi=\cos \theta, x=\cos \eta$ for some $0 \leq \theta, \eta \leq \pi$ and applying the substitution $y=\cos \phi$, we obtain

$$
\begin{aligned}
& \left|\Phi_{j} * \psi_{\xi}(x)\right| \\
& \quad \leq c 2^{j 3 / 2} \mathcal{W}\left(2^{j} ; x\right)^{-1 / 2} \int_{0}^{\pi} \frac{w(\cos \phi) \sin \phi}{\mathcal{W}\left(2^{j} ; \cos \phi\right)\left(1+2^{j}|\eta-\phi|\right)^{\sigma}\left(1+2^{j}|\theta-\phi|\right)^{\sigma}} d \phi \\
& \quad \leq c 2^{j 3 / 2} \mathcal{W}\left(2^{j} ; x\right)^{-1 / 2} \int_{0}^{\pi} \frac{1}{\left(1+2^{j}|\eta-\phi|\right)^{\sigma}\left(1+2^{j}|\theta-\phi|\right)^{\sigma}} d \phi \\
& \quad \leq c 2^{j / 2} \mathcal{W}\left(2^{j} ; x\right)^{-1 / 2}\left(1+2^{j}|\eta-\theta|\right)^{-\sigma}
\end{aligned}
$$


where we used the inequality

$$
2^{j} \int_{\mathbb{R}} \frac{1}{\left(1+2^{j}|\eta-\phi|\right)^{\sigma}\left(1+2^{j}|\theta-\phi|\right)^{\sigma}} d \phi \leq c\left(1+2^{j}|\eta-\theta|\right)^{-\sigma} .
$$

For the proof of Lemma 4.9 we will need this lemma:

Lemma 9.2. Let $P \in \Pi_{2^{j}}, j \geq 0$ and $\xi \in \mathcal{X}_{j}$. Suppose $x_{1}, x_{2} \in[-1,1]$ and $d\left(x_{\nu}, \xi\right) \leq c_{\star} 2^{-j}, \nu=1,2$. Then for any $\sigma>0$,

$$
\left|P\left(x_{1}\right)-P\left(x_{2}\right)\right| \leq c_{\sigma} 2^{j} d\left(x_{1}, x_{2}\right) \sum_{\eta \in \mathcal{X}_{j}} \frac{|P(\eta)|}{\left(1+2^{j} d(\xi, \eta)\right)^{\sigma}},
$$

where $c_{\sigma}>0$ depends only on $\sigma, \alpha, \beta$, and $c_{\star}$.

Proof. Let $P \in \Pi_{2^{j}}$. Suppose $L_{2^{j}}(x, y)$ is the reproducing kernel from Lemma 2.5 with $n=2^{j}$. Then $L_{2^{j}} * P=P$. Since $L_{2^{j}}(x, \cdot) P(\cdot) \in \Pi_{2^{j+2}}$, using the fact that the quadrature $(2.14)$ is exact for all polynomials from $\Pi_{2^{j+2}}$ we get

$$
P(x)=\int_{-1}^{1} L_{2^{j}}(x, y) P(y) w(y) d y=\sum_{\eta \in \mathcal{X}_{j}} \mathrm{c}_{\eta} L_{2^{j}}(x, \eta) P(\eta), \quad x \in[-1,1] .
$$

Recall that $\mathrm{c}_{\eta} \sim 2^{-j} \mathcal{W}\left(2^{j} ; \eta\right)$. Now, using Theorem 2.2 we obtain, for $x_{1}, x_{2} \in[-1,1]$ with $d\left(x_{\nu}, \xi\right) \leq c_{\star} 2^{-j}, \nu=1,2$,

$$
\begin{aligned}
\left|P\left(x_{1}\right)-P\left(x_{2}\right)\right| & =\left|\int_{-1}^{1}\left[L_{2^{j}}\left(x_{1}, y\right)-L_{2^{j}}\left(x_{2}, y\right)\right] P(y) w(y) d y\right| \\
& \leq \sum_{\eta \in \mathcal{X}_{j}}\left|c_{\eta}\right|\left|L_{2^{j}}\left(x_{1}, \eta\right)-L_{2^{j}}\left(x_{1}, \eta\right)\right||P(\eta)| \\
& \leq c 2^{j} d\left(x_{1}, x_{2}\right) \sum_{\eta \in \mathcal{X}_{j}}\left(\frac{\mathcal{W}\left(2^{j} ; \eta\right)}{\mathcal{W}\left(2^{j} ; \xi\right)}\right)^{1 / 2} \frac{|P(\eta)|}{\left(1+2^{j} d(\xi, \eta)\right)^{\sigma}} \\
& \leq c 2^{j} d\left(x_{1}, x_{2}\right) \sum_{\eta \in \mathcal{X}_{j}} \frac{|P(\eta)|}{\left(1+2^{j} d(\xi, \eta)\right)^{\sigma-\max \{\alpha, \beta\}-1 / 2}}
\end{aligned}
$$

where for the last inequality we used (2.22). Since $\sigma>0$ can be arbitrarily large the result follows.

Proof of Lemma 4.9. Clearly $a_{\xi} \leq b_{\xi}+d_{\xi}$, where

$$
d_{\xi}:=\max \left\{\left|P\left(x_{1}\right)-P\left(x_{2}\right)\right|: x_{1} \in I_{\xi}, d\left(x_{1}, x_{2}\right) \leq c_{2} 2^{-(j+r)}\right\},
$$

and $c_{2}$ is the constant appearing in (2.18). By Lemma 9.2 it follows that

$$
d_{\xi} \leq c 2^{-r} \sum_{\eta \in \mathcal{X}_{j}} \frac{|P(\eta)|}{\left(1+2^{j} d(\xi, \eta)\right)^{\sigma}}, \quad \xi \in \mathcal{X}_{j}
$$


Then recalling the definition of $d_{\xi}^{*}$ in $(4.16)$ we infer that

$$
\begin{aligned}
d_{\xi}^{*} & \leq c 2^{-r} \sum_{w \in \mathcal{X}_{j}} \sum_{\eta \in \mathcal{X}_{j}} \frac{|P(\eta)|}{\left(1+2^{j} d(w, \eta)\right)^{\sigma}\left(1+2^{j} d(\xi, w)\right)^{\sigma}} \\
& \leq c 2^{-r} \sum_{\eta \in \mathcal{X}_{j}} \frac{|P(\eta)|}{\left(1+2^{j} d(\eta, \xi)\right)^{\sigma}} \leq c 2^{-r} a_{\xi}^{*},
\end{aligned}
$$

where for the second inequality we switched the order of summation and used the simple fact that for $\sigma>1$,

$$
\sum_{w \in \mathcal{X}_{j}} \frac{1}{\left(1+2^{j} d(w, \eta)\right)^{\sigma}\left(1+2^{j} d(\xi, w)\right)^{\sigma}} \leq c \frac{1}{\left(1+2^{j} d(\eta, \xi)\right)^{\sigma}} .
$$

Consequently, $a_{\xi}^{*} \leq b_{\xi}^{*}+d_{\xi}^{*} \leq b_{\xi}^{*}+c 2^{-r} a_{\xi}^{*}$ with $c>0$ independent of $r$. Choosing $r$ sufficiently large we obtain $a_{\xi}^{*} \leq c b_{\xi}^{*}$. The estimate in the other direction is trivial.

Proof of Lemma 4.10. We may assume that $\alpha \geq \beta$. Fix $\xi \in \mathcal{X}_{j}$ and define $Y_{0}:=\left\{\eta \in \mathcal{X}_{j}: d(\eta, \xi) \leq c_{2} 2^{-j}\right\}$ and

$$
Y_{m}:=\left\{\eta \in \mathcal{X}_{j}: c_{2} 2^{-j+m-1}<d(\eta, \xi) \leq c_{2} 2^{-j+m}\right\}, \quad m \geq 1,
$$

where $c_{2}>0$ is from (2.18). Using (2.18) we have $\# Y_{m} \leq c 2^{m}$. Also, let $J_{m}:=B_{\xi}\left(c_{2}\left(2^{m}+1\right) 2^{-j}\right)=\left\{x \in[-1,1]: d(\eta, \xi) \leq c_{2}\left(2^{m}+1\right) 2^{-j}\right\}, \quad m \geq 0$. Evidently, $J_{m}$ is an interval and $I_{\eta} \subset J_{m}$ if $\eta \in Y_{\nu}, 0 \leq \nu \leq m$.

We next show that

$$
\mu\left(J_{m}\right) \leq c 2^{m(4 \alpha+3)} \mu\left(I_{\eta}\right) \quad \text { for all } \eta \in Y_{m} .
$$

Suppose $\xi \in[0,1]$; the case $\xi \in[-1,0]$ is the same. Let $J_{m}=:\left[y_{1}, y_{2}\right]$ and choose $\phi_{1}, \phi_{2} \in[0, \pi]$ so that $y_{1}=: \cos \phi_{1}$ and $y_{2}=: \cos \phi_{2}\left(\phi_{1}>\phi_{2}\right)$. Exactly as in the proof of Lemma 2.7,

$$
\mu\left(J_{m}\right)=\int_{J_{m}} w(y) d y \leq c\left(\phi_{1}-\phi_{2}\right) \phi_{1}^{2 \alpha+1} \leq c 2^{-j+m} \mathcal{W}\left(2^{j} ; y_{1}\right),
$$

and by $(2.22)$,

$$
\begin{aligned}
\mu\left(J_{m}\right) & \leq c 2^{-j+m} \mathcal{W}\left(2^{j} ; \xi\right)\left(1+2^{j} d\left(y_{1}, \xi\right)\right)^{2 \alpha+1} \\
& \leq c 2^{-j+(2 \alpha+2) m} \mathcal{W}\left(2^{j} ; \xi\right) .
\end{aligned}
$$

On the other hand, using again (2.22) we get

$$
\begin{aligned}
\mu\left(I_{\eta}\right) & \geq c 2^{-j} \mathcal{W}\left(2^{j} ; \eta\right) \geq c 2^{-j} \mathcal{W}\left(2^{j} ; \xi\right)\left(1+2^{j} d(\eta, \xi)\right)^{-2 \alpha-1} \\
& \geq c 2^{-j-(2 \alpha+1) m} \mathcal{W}\left(2^{j} ; \xi\right) .
\end{aligned}
$$

Combining this with (9.20) gives (9.19). 
Let $\varrho:=\max \{0,1-1 / t\}<1$. Using Hölder's inequality if $t>1$ and the $t$-triangle inequality if $0<t \leq 1$, we have

$$
\begin{aligned}
b_{\xi}^{*} & =\sum_{\eta \in \mathcal{X}_{j}} \frac{\left|b_{\eta}\right|}{\left(1+2^{j} d(\eta, \xi)\right)^{\sigma}} \leq c \sum_{m \geq 0} 2^{-m \sigma} \sum_{\eta \in Y_{m}}\left|b_{\eta}\right| \\
& \leq c \sum_{m \geq 0} 2^{-m(\sigma-\varrho)}\left(\sum_{\eta \in Y_{m}}\left|b_{\eta}\right|^{t}\right)^{1 / t} .
\end{aligned}
$$

We next use (9.19) to obtain, for $x \in I_{\xi}$,

$$
\begin{aligned}
b_{\xi}^{*} & =c \sum_{m \geq 0} 2^{-m(\sigma-1)}\left(\int_{-1}^{1}\left[\sum_{\eta \in Y_{m}}\left|b_{\eta}\right| \mu\left(I_{\eta}\right)^{-1 / t_{1}} \mathbb{1}_{I_{\eta}}(x)\right]^{t} w(x) d x\right)^{1 / t} \\
& \leq c \sum_{m \geq 0} 2^{-m(\sigma-1)}\left(\frac{1}{\mu\left(J_{m}\right)} \int_{J_{m}}\left[\sum_{\eta \in Y_{m}}\left(\frac{\mu\left(J_{m}\right)}{\mu\left(I_{\eta}\right)}\right)^{1 / t}\left|b_{\eta}\right| \mathbb{1}_{I_{\eta}}(x)\right]^{t} w(x) d x\right)^{1 / t} \\
& \leq c \sum_{m \geq 0} 2^{-m(\sigma-1-(4 \alpha+3) / t)}\left(\frac{1}{\mu\left(J_{m}\right)} \int_{J_{m}}\left[\sum_{\eta \in Y_{m}}\left|b_{\eta}\right| \mathbb{1}_{I_{\eta}}(x)\right]^{t} w(x) d x\right)^{1 / t} \\
& \leq c \mathcal{M}_{t}\left(\sum_{w \in \mathcal{X}_{j}}\left|b_{w}\right| \mathbb{1}_{I_{\omega}}\right)(x) \sum_{m \geq 0} 2^{-m(\sigma-1-(4 \alpha+3) / t)} \\
& \leq c \mathcal{M}_{t}\left(\sum_{w \in \mathcal{X}_{j}}\left|b_{w}\right| \mathbb{1}_{I_{\omega}}\right)(x)
\end{aligned}
$$

where for the last inequality we used the fact that $\sigma>(4 \alpha+3) / t+1$.

Proof of Lemma 6.5. For $\xi \in \mathcal{X}_{j}$, we set $a_{\xi}:=\max _{x \in I_{\xi}}|P(x)|, m_{\xi}:=$ $\min _{x \in I_{\xi}}|P(x)|$ and

$$
b_{\xi}:=\max \left\{\min _{x \in I_{w}}|P(x)|: w \in \mathcal{X}_{j+r}, I_{w} \cap I_{\xi} \neq \emptyset\right\},
$$

where $r \geq 1$ (sufficient large) is the constant from Lemma 4.9. If $0<t<p$ then

$$
\begin{aligned}
\left(\sum_{\xi \in \mathcal{X}_{j}} a_{\xi}^{p} \mu\left(I_{\xi}\right)\right)^{1 / p} & =\left\|\sum_{\xi \in \mathcal{X}_{j}} a_{\xi} \mathbb{1}_{I_{\xi}}(\cdot)\right\|_{p} \leq c\left\|\sum_{\xi \in \mathcal{X}_{j}} b_{\xi}^{*} \mathbb{1}_{I_{\xi}}(\cdot)\right\|_{p} \\
& \leq c\left\|\mathcal{M}_{t}\left(\sum_{\xi \in \mathcal{X}_{j}} b_{\xi} \mathbb{1}_{I_{\xi}}\right)(\cdot)\right\|_{p} \leq c\left\|\sum_{\xi \in \mathcal{X}_{j}} b_{\xi} \mathbb{1}_{I_{\xi}}(\cdot)\right\|_{p},
\end{aligned}
$$

where for the first inequality we used Lemma 4.9 and for the second Lemma 4.10. Also, for $\xi \in \mathcal{X}_{j}$ let $\mathcal{X}_{j+r}(\xi):=\left\{w \in \mathcal{X}_{j+r}: I_{w} \cap I_{\xi} \neq \emptyset\right\}$. Evidently, $\# \mathcal{X}_{j+r}(\xi) \leq c$. Then for $w, \eta \in \mathcal{X}_{j+r}(\xi)$ we have $d(w, \eta) \leq c(r) 2^{-j-r}$ and hence

$$
m_{w} \leq c \frac{m_{w}}{1+2^{j+r} d(w, \eta)} \leq c m_{\eta}^{*} .
$$


Therefore, for any $\xi \in \mathcal{X}_{j}$ and $\eta \in \mathcal{X}_{j+r}(\xi)$ we have $b_{\xi}=\max _{w \in \mathcal{X}_{j+r}(\xi)} m_{w} \leq$ $c m_{\eta}^{*}$ and hence

$$
b_{\xi} \mathbb{1}_{I_{\xi}} \leq \sum_{\eta \in \mathcal{X}_{j+r}(\xi)} m_{\eta}^{*} \mathbb{1}_{I_{\eta}} .
$$

Using this in (9.21) we get

$$
\begin{aligned}
\left(\sum_{\xi \in \mathcal{X}_{j}} a_{\xi}^{p} \mu\left(I_{\xi}\right)\right)^{1 / p} & \leq c\left\|\sum_{\eta \in \mathcal{X}_{j+r}} m_{\eta}^{*} \mathbb{1}_{I_{\eta}}(\cdot)\right\|_{p} \leq c\left\|\mathcal{M}_{t}\left(\sum_{\eta \in \mathcal{X}_{j+r}} m_{\eta} \mathbb{1}_{I_{\eta}}\right)(\cdot)\right\|_{p} \\
& \leq c\left\|\sum_{\eta \in \mathcal{X}_{j+r}} m_{\eta} \mathbb{1}_{I_{\eta}}(\cdot)\right\|_{p} \leq c\|P\|_{p},
\end{aligned}
$$

which completes the proof.

\section{References}

[1] G. Brown and F. Dai, Approximation of smooth functions on compact two-point homogeneous spaces, J. Funct. Anal. 220 (2005), 401-423.

[2] R. Coifman and G. Weiss, Extensions of Hardy spaces and their use in analysis, Bull. Amer. Math. Soc. 83 (1977), 569-645.

[3] W. Connett and A. Schwartz, A multiplier theorem for Jacobi expansions, Studia Math. 52 (1975), 234-261.

[4] Z. Ditzian and V. Totik, Moduli of Smoothness, Springer, 1987.

[5] C. L. Frenzen and R. Wong, A uniform asymptotic expansion of the Jacobi polynomials with error bounds, Canad. J. Math. 37 (1985), 979-1007.

[6] M. Frazier and B. Jawerth, Decomposition of Besov Spaces, Indiana Univ. Math. J. 34 (1985), 777-799.

[7] —, - A discrete transform and decompositions of distributions, J. Funct. Anal. 93 (1990), 34-170.

[8] M. Frazier, B. Jawerth, and G. Weiss, Littlewood-Paley Theory and the Study of Function Spaces, CBMS Reg. Conf. Ser. Math. 79, Amer. Math. Soc., 1991.

[9] G. Kyriazis, P. Petrushev, and Y. Xu, Decomposition of weighted Triebel-Lizorkin and Besov spaces on the ball, Proc. London Math. Soc., to appear.

[10] B. Muckenhoupt and E. Stein, Classical expansions and their relation to conjugate harmonic functions, Trans. Amer. Math. Soc. 118 (1965), 17-92.

[11] F. Narcowich, P. Petrushev, and J. Ward, Decomposition of Besov and TriebelLizorkin spaces on the sphere, J. Funct. Anal. 238 (2006), 530-564.

[12] P. Nevai, Orthogonal Polynomials, Mem. Amer. Math. Soc. 18 (1979).

[13] P. Petrushev and Y. Xu, Localized polynomial frames on the interval with Jacobi weights, J. Fourier Anal. Appl. 11 (2005), 557-575.

[14] - - - Localized polynomial frames on the ball, Constr. Approx. 27 (2008), 121-148.

[15] J. Peetre, New Thoughts on Besov Spaces, Duke Univ. Math. Ser., Durham, NC, 1976.

[16] T. Runst and W. Sickel, On strong summability of Jacobi-Fourier-expansions and smoothness properties of functions, Math. Nachr. 99 (1980), 77-85.

[17] E. Stein, Harmonic Analysis: Real-Variable Methods, Orthogonality, and Oscillatory Integrals, Princeton Univ. Press, Princeton, NJ, 1993. 
[18] G. Szegő, Orthogonal Polynomials, 4th ed., Amer. Math. Soc. Colloq. Publ. 23, Providence, 1975.

[19] H. Triebel, Theory of Function Spaces, Monogr. Math. 78, Birkhäuser, Basel, 1983.

Department of Mathematics and Statistics

University of Cyprus

1678 Nicosia, Cyprus

E-mail: kyriazis@ucy.ac.cy

Department of Mathematics

University of Oregon

Eugene, OR 97403, U.S.A.

E-mail: yuan@math.uoregon.edu
Department of Mathematics

University of South Carolina

Columbia, SC 29208, U.S.A.

E-mail: pencho@math.sc.edu

and

Institute of Mathematics and Informatics

Bulgarian Academy of Sciences

Received October 21, 2006

Revised version March 10, 2008 